\title{
Volatility Widens Inequality. Could Aid and Remittances
}

\section{Help?}

Lisa Chauvet: IRD, LEDa, PSL, UMR DIAL, Université Paris Dauphine, FERDI

MARIN FERry : Université Paris Dauphine, LEDa, PSL, UMR DIAL

Patrick Guillaumont : FERDI

Sylviane Guillaumont Jeanneney : Université d'Auvergne CERDI

Sampawende J.-A. Tapsoba: International Monetary Fund

LAURENT WAGNER: FERDI

\begin{abstract}
We analyse the relationship between income volatility and inequality and the conditional role played by aid and remittances. Using a panel of 142 countries for the period 1973 to 2012, we find that income volatility has an adverse impact on inequality, and that the poorest people are the most exposed to these fluctuations. However, while aid and remittances do not seem to have a clear direct impact on inequality, we uncover robust evidence which suggests that aid helps to dampen the negative effects of volatility on the distribution of income, while remittances do not.
\end{abstract}

Keywords : Volatility, Inequality, Aid, and Remittances

JEL classification : $\mathrm{F}_{24}, \mathrm{~F}_{35}, \mathrm{O}_{15}$

The authors are grateful to Maddalena Agnoli and Sosso Feindouno for their assistance at the early stage of this work. Financial support from the DFID-ESRC Growth Research Programme, under Grant No. ES/L012022/1, is gratefully acknowledged. The views expressed in this paper are those of the authors.

The paper, and other contributions to the project, can be downloaded at:

- http://www.socialsciences.manchester.ac.uk/subjects/economics/ourresearch/cgbcr/esrc-dfid-project/

- http://www.ferdi.fr/en/programme-project/financial-volatility 


\section{Introduction}

The adverse impact of volatility on developing countries' performance has been documented at length. After several decades of analysis of the impact of export instability on developing countries, income volatility has been shown to have a negative impact on economic growth (Ramey and Ramey, 1995; Hnatkovska and Loayza, 2005). Exogenous sources of instability, due to external trade shocks or natural disasters, and the volatility they induce, are significant factors which have the effect of lowering average income growth. The effect is particularly severe in countries which are highly exposed to exogenous fluctuations. It is now well established that income volatility has harmful consequences for development (see Guillaumont 2006, 2009 for a comprehensive review). Numerous papers have shown the negative effect on the average growth of income of both income growth instability (Ramey and Ramey, 1995; Hnatkovska and Loayza, 2005; Norrbin and Yigit, 2005), and specific exogenous instabilities, especially export instability in Africa (Guillaumont et al., 1999). The negative effects of income volatility on growth result both from uncertainty and risk-aversion (ex ante effect), and from asymmetric responses to positive and negative shocks (ex post effect).

However, not only does volatility affect the size of the pie, it also impacts its distribution. Volatility increases income inequality, making growth less favourable to the poor. The fact that income reductions disproportionately affect the poorest households, combined with the asymmetric impacts of recoveries and reductions on different categories of the population, implies that output volatility is associated with higher inequality. Poor people are more vulnerable to volatility than rich people. They have less diversified sources of income, are less qualified, and are less mobile between sectors and geographic areas (Agénor, 2004; Laursen and Mahajan, 2005). Likewise, they have little access to credit and insurance markets, and depend more on state aid and social services (Guillaumont Jeanneney and Kpodar, 2011). The poor engage in sub-optimal smoothing of income shocks for instance by investing in livestock, which is subject to lumpiness. The inability of poor people to face negative shocks results in losses of human capital, which are difficult to reverse.'

In this paper, we proceed in two steps. First, we estimate the impact of income volatility on inequality, measured either using the Gini coefficient or the income share by quintile. We next add to our specification foreign aid, then an interaction term of aid with volatility and alternately remittances, and finally an interaction term of remittances with volatility. This strategy allows us to identify the potential mitigating effect of aid and remittances (through their interactive term with income volatility), as well as their direct impact on income inequality (through their value in level, not interacted). We use the panel fixed effects and system GMM estimators. We find robust evidence suggesting that volatility increases inequality, which is in line with the literature. We also

\footnotetext{
1 e.g. Nutritional status (Dercon and Krishnan, 2000, for Ethiopia), or removing children from school (Thomas et al., 2004, for Indonesia).
} 
find that while aid does not seem to impact inequality directly, the interaction term of aid with volatility is significantly associated with a less skewed income distribution. Conversely, the results for remittances, although similar when using the fixed effects estimator, are found to be not robust with the GMM specification.

In a second step, we investigate the micro-foundations of the mitigating role of foreign aid, and also explore the other potential channel through which aid and remittances reduce inequalities, i.e. their stabilizing effect. We estimate their impact on income volatility, accounting for different levels of exports and external volatility. We find that aid dampens income volatility for countries which are open and experience significant export volatility. However, no stabilizing effect is observed for remittances. Our results suggest that aid affects inequality, both by reducing income volatility and by mitigating the adverse impact of income volatility on inequality. This effect is not seen for remittances.

This paper is structured as follows :- The relevant literature is presented in Section 2, and model and the data in Section 3. The results of the baseline estimations are presented in Section 4, and robustness checks in Section 5. Section 6 discusses the transmission channels of aid on inequality, showing the mitigating effect of aid on income volatility. Section 7 offers conclusions.

\section{Literature review}

In this paper, we explore the role played by foreign aid and remittances in the relationship between income volatility and inequality. In many developing countries, foreign aid is not the main source of public external financing, and in some of them remittances represent the largest share of their international financial inflows. For this reason, the impact of remittances has also been analysed. As shown in Figure 1, there exist various potential channels of effect between income volatility, aid or remittances, and inequality. As explained above, income volatility is likely to widen income distribution (relation 1). Foreign aid and remittances may affect income inequality in three different ways. First these external financial flows can mitigate the adverse effect of income volatility on inequality, increasing the resilience of countries facing negative income shock (relation 2). Aid is likely to reduce the positive impact of income volatility on inequality in so far as it allows more public spending in favour of the poor (safety nets or social expenditure). In the same theme, remittances can also help households dealing with unexpected negative income shocks. Second, these flows might directly impact income distribution (relation 3), although no consensus on this question has yet been reached. Third, the aid/inequality and remittances/inequality relationships can also be examined through the lens of the potential stabilizing role of aid and remittances (relation 4). 
Figure 1: Channels of effect between aid, remittances, economic volatility, and income inequality

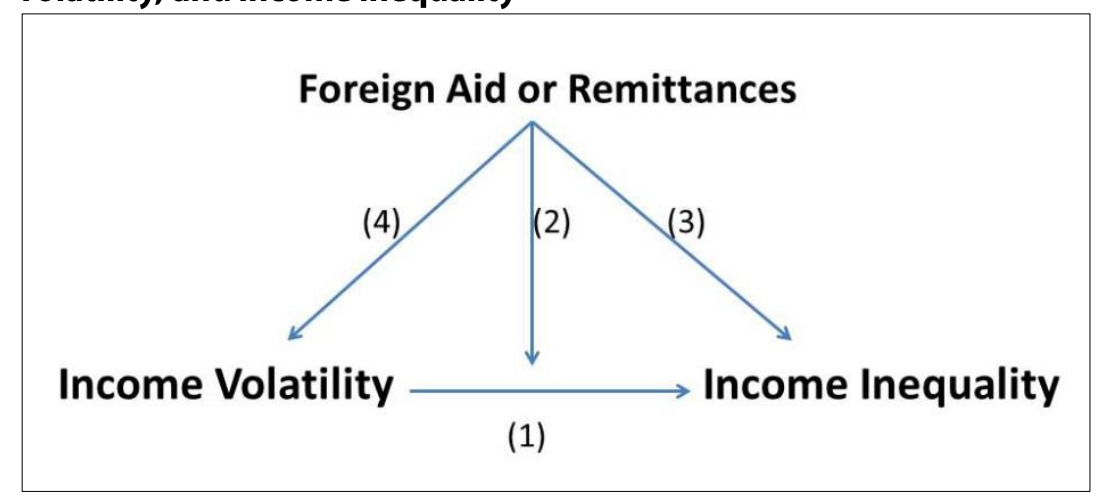

(1) Direct effect of income volatility on inequality

(2) Resilience or mitigating effect of aid or remittances

(3) Direct effect of aid or remittances on inequality

(4) Stabilizing effect of aid or remittances on income volatility

A few cross-country econometric analyses of the effects of income volatility on inequality have been performed. Laursen and Mahajan (2005) find a negative effect of income volatility on the poorest quintile, while for Breen and Garcia-Penalosa (2005) quintiles Q2 \& Q3 (rather than the poorest one, Q1) appear to be the most affected, suggesting that people who are nearly poor may become durably poor under unstable conditions. More recently Calderon and Levy Yeyati (2009) have also shown the effect of output volatility on income distribution, captured through both the Gini coefficient and the different impact by quintile. They find a non-linear impact of volatility, which depends on the level of public expenditure, considered as a mitigating factor.

Even if internal factors such as political instability or economic mismanagement explain a large part of income volatility (Raddatz, 2007), developing countries remain highly exposed to external shocks, such as high price volatility of their main export commodity. They are also exposed to natural shocks, which, given the share of the primary sector in their total income, have a big impact on their performance and their volatility. At the macroeconomic level, a significant impact can be expected from aid and/or remittances on inequality through their stabilizing effect on volatility (Guillaumont and Chauvet, 2001; Chauvet and Guillaumont, 2004, 2009; Guillaumont and Le Goff, 2010; Le Goff 2010, Guillaumont and Wagner, 2012).

To the best of our knowledge, research on the empirical relation between foreign aid and/or remittances and income inequality is still limited. While most literature focuses on aid effectiveness in terms of economic growth, only a smaller body of literature examines the effects on poverty reduction and inequality (see for instance Guillaumont and Wagner (2013) for a survey with a special focus on the interactions between aid, poverty, and income volatility). Chase-Dunn (1975) provided one of the first empirical analyses of the aid/inequality relationship, although within the very different overall framework of Marxist dependency theory, and argued that there is a positive association between aid and inequality. The question then virtually disappeared from the research agenda. In the 1990s, Boone (1996) provided a theoretical discussion of whether foreign aid 
reaches the poor, or mainly benefits political elites, and concludes that the aid/growth relationship is consistent with a model in which aid contributes to a widening of inequality, in favor of the wealthy elite. The aid/inequality relationship has been investigated more recently but still without reference to the potential mitigating role of aid. Indeed Calderón et al. (2009) examine the direct effect of foreign aid on income inequality and poverty reduction for the period 1971 to 2002, highlighting the role of public expenditure. But these authors find no robust association between aid inflows and income inequality as measured by Gini coefficients. The aid/inequality relationship has also been investigated with reference to the political institutions prevailing in the receiving countries. Bjørnskov (2010) examines the joint effects of foreign aid and democracy on income quintiles for 88 countries over the 1960-2000 period. He finds that the combination of foreign aid and democracy is associated with a higher share of income held by the upper quintile. According to his study, foreign aid leads to a more skewed income distribution in democratic developing countries, while this adverse effect is negligible in autocratic countries. He highlights some potential mechanisms to explain why aid may make income distribution more skewed in developing countries, such as rent-seeking activities induced by elections in weak democracies, or Dutch disease-like phenomena. However, using a similar data and identification strategy, Chauvet and Mesplé-Somps (2007) reach very different conclusions. They find that aid tends to increase the income share of the middle class in democracies, since aid is less likely to be captured by the elite.

The literature on the relationship between remittances and income distribution or poverty is prolific and has not reached a consensus. ${ }^{2}$ Moreover the potential mitigating effect of remittances has not been taken into account. According to the review of the literature by Rapoport and Docquier (2006), at the macro level, "there is considerable evidence that remittances (in the form of savings repatriated by return migrants) promote access to self-employment and raise investment in small businesses, and there is also evidence that remittances contribute to raise educational attainments of children in households with migrant members. However, the relationship between remittances and inequality appears to be non-monotonic: remittances seem to decrease economic inequality in communities with a long migration tradition but to increase inequality within communities at the beginning of the migration process. This is consistent with different theoretical arguments regarding the role of migration networks and/or the dynamics of wealth transmission between successive generations." It seems that the impact of remittances on income inequality depends on who migrates, i.e. on the quintile migrants occupy in income distribution in their home country (Ebeke and Le Goff, 2009).

Whether or not aid and remittances have a stabilizing role to play regarding external shocks and income volatility has also been debated. The volatility of aid has been presented as a potential source of income volatility by several authors (Bulir and Hamann, 2001, 2008; Pallage and Robe,

\footnotetext{
${ }^{2}$ See for instance Stark, Taylor,and Yitzhaki (1998); Taylor (1992); Barham and Biycher (1998); Adams and Page (2005); Le Goff (2010).
} 
2001): they argue that aid is very often more volatile than fiscal revenues and income, but also more often pro-cyclical than counter-cyclical with respect to these variables, which are not independent of aid. This finding has been challenged by Chauvet and Guillaumont (2009) who consider the evolution of aid with respect to exports. Moreover, whatever its pro- or contra-cyclical evolution, aid appears more often to have a stabilizing impact still with respect to exports (Ibid.). The same authors find that the average aid to GDP ratio lowers income volatility, while aid volatility (weighted by the aid to GDP ratio) has the opposite effect. The result is that aid may be more effective in countries exposed to strong and/or recurrent exogenous shocks. Thus, foreign aid could, in principle and in the short to medium run, benefit the poor by dampening the negative effect of income volatility. In this paper we examine whether this stabilizing effect induces lower income volatility, hence lower inequality.

The findings are similar for remittances. Combes, Ebeke and Ntsama Etoudi (2014) have shown that in low income countries, and especially in the Sub-Saharan African region where most countries are vulnerable to food price shocks, remittances and aid inflows dampen the effect of food price shocks and instability on household consumption. Similar evidence for the stabilizing effect of remittances can also be found in Bugamelli and Paternò (2011), Bouoiyour et al (2016) and De et al (2016).

According to Le Goff and Guillaumont (2010), at the country level aid flows and remittances flows exert a stabilizing impact with regard to the fluctuation of exports, as distinct from their countercyclical character. More often than the contrary, both kinds of flow appear to have stabilizing effects. Second, on a cross-country basis, aid and remittances dampen growth volatility, while at a country level, remittances appear to dampen the instability of exports more often than aid; on a cross-country basis, aid lowers growth volatility more than remittances.

\section{Empirical section}

\subsection{Methodology}

We estimate an inequality equation in which we include income volatility and our variables which represent external financing (either foreign aid or remittances). In order to assess the mitigating effect of these flows on income volatility we estimate the following equation:

$$
\begin{aligned}
& I_{N E Q_{i, t}=\lambda I N E Q_{i, t-5}+\alpha V O L A T I L I T Y_{i, f(t,-5)}+\beta E X T . F I N A N C I N G},((t, t-5) \\
& +\delta \text { EXT.FINANCING } G_{i,(t, t-5)} \times \operatorname{VOLATILITY}_{i,(t, t-5)}+\gamma X_{i,(t, t-5)}+\mu_{i}+\tau_{t}+\varepsilon_{i, t}
\end{aligned}
$$

where $I N E Q_{i, t}$ is the measure of inequality of country $i$, in year $t$. We include the lag in $t-5$ of $I N E Q_{i, t-5}$ on the right-hand side in order to account for a catching up effect. The model includes EXT.FINANCING ${ }_{(t, t,-5)}$ which represents foreign aid or remittances, and control variables $X_{i,(t, t-5),}$ averaged over $t$ and $t-5$. Volatility is also measured over this time span. In order to account for 
country unobservable heterogeneity, we include country fixed effects, $\mu_{i}$. We also include period fixed effects, $\tau_{t}$, to account for global business cycles.

\subsection{Data}

\section{Measure of Inequality}

To facilitate cross-country comparisons, several attempts have been made to produce harmonized inequality series. We list here four of the most recent and up-to-date attempts. The World Bank initiative, "Povcalnet", from Chen and Ravallion (2008) proposes harmonized and interpolated series inequality measures (Gini index and income deciles) from 1981 to 2011 on a 3-year interval for the majority of developing countries. Data are retrieved from individual household surveys, and made comparable across countries and time. However, most of the data points are estimates interpolated from the most recent surveys available which for the poorest countries might lead to bias. The second World Bank initiative, "World Income Distribution (WYD)", from Milanovic (2013), proposes average per capita income of various fractiles of population expressed in domestic currency units, from 1988 to 2005 on a 5-year interval. Income or consumption data from surveys not conducted in the benchmark years $(1988,1993,1998,2002$, and 2005) are adjusted by simply assuming an unchanged distribution and deflating/inflating incomes by countries' Consumer Price Index between the actual survey year and the benchmark year.

The "World Income Inequality Database (WIID)", is produced by the United Nations University WIDER, following the previous work of Deninger and Squire (1996). It lists, aggregates, compares, and rates the results of all available income and consumption surveys from 1960 to 2012. The figures are ordered and labelled to be made comparable across time and countries, but not interpolated; which means that the coverage of the database is more limited than the others. Compared to the Povcalnet database, the WIID also includes data derived from low quality surveys in terms of design and coverage. The use of these data points can increase the coverage of the database but comes at the expense of precision. ${ }^{3}$

Following the recommendations of Atkinson and Brandolini $(2001,2009)$ we chose to work with the WIID database as it includes a lot of additional information which allows the selection of consistent inequality estimates. It also allows working without interpolated data that might distort the end results. As a secondary data compilation about income inequality, the World Income Inequality Database (WIID) suffers from many caveats that must be clearly addressed to provide consistent results. The principal issue when building cross-section time series on income inequality

\footnotetext{
${ }^{3}$ It is also worth mentioning the work of Stolt (2014), who, building on the WIID, proposed an interpolated version of the dataset, the "Standardized World Income Inequality Database (SWIID)". The SWIID provides comparable estimates of the Gini index for 174 countries from 1960 to 2012, as well as measures of absolute and relative redistribution. Data points are fully interpolated and should be used cautiously.
} 
mainly stems from the lack of comparability of the underlying household surveys and the coverage and definitions they use, notably in terms of whether the data derive from a survey based on consumption/expenditure or income/earnings. Due to data availability, we favour income rather than consumption. ${ }^{4}$ Consumption data were used only when no income data was available. In those cases a dummy variable, CONSUMPTION ${ }_{i, t}$ is included in the model to control for the discrepancy in the measure of inequality. We also discarded data points that did not cover the whole population, that is to say urban-only or rural-only surveys. The WIID dataset includes both Gini and quantile data. In the regressions, we use both sources of information.

\section{Measure of Volatility}

In many studies, income volatility has been measured as the standard deviation of the growth rate of real income or income per capita Ramey and Ramey, 1995; Breen and Garcia-Peñaloza, 2005; Raddatz, 2007; Calderon and Levy Yeyati, 2009). Many alternative measures exist, which account better for the cyclical characteristics of the output. We favour a method that measures economic volatility as the standard deviation of the cycle (relative to the trend) of output, because this measure is based on a less restrictive formalization of the process underlying the change in the trend of economic series. The cycle of output is the residual of an econometric regression accounting for a time trend as well as a stochastic trend. For each country we therefore estimate an equation of the following form:

$y_{t}=\alpha \tau_{t}+\beta y_{t-1}+\varepsilon_{t}$

where $\tau_{t}$ is a time trend, $y_{t}$ is income per capita in year $t$ and, $y_{t-1}$ is income per capita in year $t-1$. Volatility of income is then measured as the standard deviation of $\varepsilon_{t} / \hat{y}_{t}$ over five years.

As a robustness check, we will also consider the simpler approach used by Calderon and Levy Yeyati (2009) who measure output volatility using the standard deviation of per capita GDP growth.

\section{Aid and control variables}

We measure foreign aid, $A I D_{i,(t, t-5),}$ using the ratio of the net disbursements of official development assistance provided by the OECD-DAC to GDP. In order to maximize sample size, we choose to keep non-aid recipient countries in the sample. However, we cannot simply use $\log \left(A I D_{i,(t,-5)-5)}\right)$ as the aid variable without losing the observations with zero aid, the log of which is undefined. Following Wagner (2003), the aid variable becomes $0.0001+A I D_{i, t, t-5)}$ and is then transformed using $\log \left(\max \left\{0.0001, A I D_{i, t, t-5)}\right\}\right.$. It is then complemented in the regressions by a "no aid" dummy variable,

\footnotetext{
${ }^{4}$ Measures based on consumption data reflect more accurately income distribution, but would restrict our sample too much.
} 
which equals 1 when $A I D_{i,(t, t-5)}=0.0001$. Following Calderon and Levy Yeyati (2009) and Bjørnskov (2010), we control for income per capita and its square, the gross secondary school enrolment rate, and the ratio of public spending to GDP. Following Bjørnskov (2010), we also introduce the ratio of rural population to total population, the rate of inflation, and population growth rate. All variables (except population growth and income volatility) are in logarithm. Aid and the control variables are averaged over five-year periods, for the period 1973 to 2012. Our sample of countries includes a maximum of 142 countries. Remittances and all control variables are taken from the World Development Indicators.

Table 1 presents the summary statistics for our sample of countries. The 142 countries included in the sample are displayed in Table A0 in Appendix. Our sample of countries displays a rather unequal distribution of income, with an average Gini index of 38 and an income share of the lowest quintiles $(\mathrm{Q} 1+\mathrm{Q} 2)$ that is only $40 \%$ of the highest quintile income share. Aid represents, on average $5.9 \%$ of the GDP. The sample of 142 countries shows an average income volatility of around $0.35 \%$, which hides a large heterogeneity, with Sub-Saharan Africa having higher levels of volatility $(0.7 \%)$, and Latin America and Asia having a lower level (0.3\%).

Table 1: Summary statistics

\begin{tabular}{lccccc}
\hline VARIABLES & $\begin{array}{c}(1) \\
\text { No of } \\
\text { Observations }\end{array}$ & $\begin{array}{c}(2) \\
\text { Mean }\end{array}$ & $\begin{array}{c}(3) \\
\text { Standard } \\
\text { Deviation }\end{array}$ & $\begin{array}{c}(4) \\
\text { Minimum }\end{array}$ & $\begin{array}{c}\text { Maximum } \\
\text { Gini index }\end{array}$ \\
Income share Q1 & 520 & 38.37 & 9.57 & 19.40 & 69.20 \\
Income share Q2 & 475 & 6.39 & 2.22 & 0.96 & 12.03 \\
Q1/Q5 & 473 & 10.92 & 2.51 & 3.03 & 15.90 \\
(Q1+Q2)/Q5 & 475 & 0.152 & 0.074 & 0.013 & 0.411 \\
Income volatility & 473 & 0.408 & 0.171 & 0.055 & 0.930 \\
Ratio of Aid to GDP & 520 & 0.348 & 0.842 & 0.010 & 13.03 \\
GDP per capita 2005 constant USD & 330 & 5.87 & 8.52 & -0.19 & 52.82 \\
Ratio of Rural population to Total & 520 & 11,97 & 15,500 & 133.3 & 81,445 \\
population & 520 & 42.76 & 22.28 & 0.654 & 95.01 \\
Population Growth & & & & & 12.39 \\
Ratio of Government expenditure to GDP & 520 & 1.33 & 1.24 & -1.84 & 84.06 \\
Inflation rate & 520 & 16.09 & 6.23 & 4.20 & 847.4 \\
Secondary school enrolment rate (gross) & 520 & 16.27 & 55.85 & 0.03 & 155.2 \\
Polity IV index & 520 & 73.11 & 31.12 & 5.32 & 10 \\
Ratio of Remittances to GDP & 494 & 5.163 & 5.926 & -10 & 87.91 \\
& 470 & 3.192 & 6.869 & 0.0002 & 142 \\
\hline Number of countries & & & & & 142 \\
\hline
\end{tabular}

Authors' calculations for a sample of 142 countries 


\section{Results}

First, we check whether the impact of income volatility on inequality is in line with the literature (Breen and Garcia-Penalosa, 2005; Calderon and Levy Yeyati, 2009). Table 2 presents the estimation results for equation (1) using simple fixed effects estimators but without the introduction of aid or remittances and their interaction term with income volatility. From column (1) using the Gini index as a dependent variable and columns (2) to (5) using quintiles of income shares as well as ratios with respect to the highest quintile, it clearly appears that economic volatility has a strong and significant impact on inequality, and that this adverse impact is bigger on the poorest. Moreover, comparing the coefficient of volatility in regressions (2) and (4), it also appears that income volatility affects disproportionately negatively the poorest segments of the population.

When introduced in Table 3, aid does not seem to impact the level of inequality in any way. ${ }^{5}$ However, the interaction variable of aid $\mathrm{x}$ income volatility influences inequality significantly. When considering the absolute income shares of the poorest quintile, as well as the ratios of the income shares of the two poorest quintiles relative to the income share of the highest quintile, the interaction term of aid $x$ income volatility is significantly positive. These results are consistent with the hypothesis that aid helps to dampen the adverse effects of volatility.

Turning next to the effect of remittances on inequality, the results show that the level of remittances negatively affects the income share of the poorest. We also find that the interaction with income volatility is significant, positive, and in all cases tends to offset the negative effect of the level of remittances. The findings for remittances thus also show a kind of mitigating effect when income volatility is high.

However, the fixed effects estimator suffers from many caveats. The first one is that it does not control for the likely endogeneity of our variables for external financing and income volatility with inequality. The second is that it does not control for the high level of persistence in the data captured by INEQ $Q_{i, t-5}$. To tackle these issues, we next use a dynamic GMM system estimator and include a lagged dependent variable on the right-hand side of the model. This also allows us to control for endogeneity by using lags of the right-hand side variables as instruments. More specifically, we assume that volatility, aid, remittances, and the interaction terms of aid and remittances with volatility are endogenous. These variables are instrumented using their lags and difference in lags from $t-2$ to $t-3$ in order to keep the number of instruments below the number of countries (Roodman, 2009).

The results using both the Gini index and the quintile income shares are displayed in Table 4 from columns (1) to (5) for foreign aid, and from columns (6) to (10) for remittances. The results relative

\footnotetext{
${ }^{5}$ When the aid variable is introduced without the interaction term aid $x$ volatility, the coefficient of the aid variable is not significantly different from zero. Results available from the authors upon request.
} 
to aid and volatility are in line with the earlier results. When volatility is high, aid reduces inequality by dampening its adverse effects on inequality. Furthermore, it also appears that the mitigating effect of aid on inequality is focused on the poorest quintiles, thereby increasing their income shares both in level and with respect to the richest quintile.

However, when tackling endogeneity with GMM estimators, the results for remittances are no longer robust, thus casting doubts about the mitigating effects of personal transfers in the home country.

Finally, in Table 5 we add the ratio of remittances to GDP and its interaction with income volatility into the equation alongside foreign aid and its relative interaction term. It appears that the results relative to remittances are still not significant, this is as expected since the literature suggests that the impact of remittances differs a lot between countries. However, even with the introduction of remittances in the same regression, we observe that the results for aid remain consistent with our baseline estimations in Tables 3 and 4, although being less significant.

Table 2: Income inequality (Gini and Income share quintiles) and volatility, panel fixed effects, 19732012, 5-year periods.

\begin{tabular}{lccccc}
\hline Fixed effects estimator & 1 & 2 & 3 & 4 & 5 \\
\hline $\begin{array}{l}\text { Dependent variables } \\
\text { (in log) }\end{array}$ & Gini & Q1 & Q2 & Q1/Q5 & $(\mathrm{Q} 1+\mathrm{Q} 2) / \mathrm{Q} 5$ \\
\hline GDP per capita volatility & $0.052^{* * *}$ & $-0.079^{* * *}$ & $-0.066^{* * *}$ & $-0.123^{* * *}$ & $-0.116^{* * *}$ \\
& $(0.015)$ & $(0.025)$ & $(0.013)$ & $(0.033)$ & $(0.024)$ \\
\hline GDP per capita (in log) & $0.514^{*}$ & $-1.135^{* * *}$ & $-0.573^{* * *}$ & $-1.563^{* * *}$ & $-1.172^{* * *}$ \\
& $(0.262)$ & $(0.405)$ & $(0.219)$ & $(0.522)$ & $(0.398)$ \\
GDP per capita squared (in log) & $-0.032^{* *}$ & $0.072^{* * *}$ & $0.035^{* *}$ & $0.100^{* * *}$ & $0.074^{* * *}$ \\
& $(0.015)$ & $(0.026)$ & $(0.013)$ & $(0.034)$ & $(0.025)$ \\
Population growth & -0.011 & 0.018 & 0.014 & 0.023 & 0.021 \\
& $(0.018)$ & $(0.039)$ & $(0.021)$ & $(0.049)$ & $(0.037)$ \\
Rural population (in log) & -0.023 & 0.080 & 0.018 & 0.091 & 0.048 \\
& $(0.037)$ & $(0.059)$ & $(0.032)$ & $(0.075)$ & $(0.056)$ \\
Inflation (in log) & 0.010 & -0.017 & 0.001 & -0.020 & -0.007 \\
& $(0.011)$ & $(0.024)$ & $(0.010)$ & $(0.030)$ & $(0.020)$ \\
Secondary school enrolment & $-0.118^{* *}$ & $0.284^{* * *}$ & $0.182^{* * *}$ & $0.395^{* * *}$ & $0.322^{* * *}$ \\
(gross, in log) & $(0.055)$ & $(0.102)$ & $(0.061)$ & $(0.136)$ & $(0.109)$ \\
Ratio of Government expenditure & 0.062 & $-0.221^{* *}$ & $-0.144^{* * *}$ & $-0.274^{* *}$ & $-0.241^{* *}$ \\
to GDP (in log) & $(0.048)$ & $(0.102)$ & $(0.051)$ & $(0.130)$ & $(0.093)$ \\
Consumption dummy & -0.029 & 0.064 & 0.031 & 0.078 & 0.049 \\
& $(0.028)$ & $(0.057)$ & $(0.035)$ & $(0.076)$ & $(0.062)$ \\
\hline R-squared & 0.145 & 0.137 & 0.161 & 0.148 & 0.168 \\
\hline Number of observations & 520 & 477 & 475 & 477 & 475 \\
Number of countries & 142 & 140 & 140 & 140 & 140 \\
\hline
\end{tabular}

Notes: Robust standard errors in parentheses, $+p<0.15,{ }^{*} p<0.10,{ }^{* *} p<0.05,{ }^{* *} p<0.01$. Each specification includes period dummies and a constant.

Sources: Authors' calculations based on UNU-WIDER, World Bank and OECD data. 
Table 3: Income inequality (Gini and Income share quintiles), volatility, aid and remittances, panel fixed effects, 1973-2012, 5-year periods.

\begin{tabular}{|c|c|c|c|c|c|c|c|c|c|c|}
\hline $\begin{array}{l}\text { Fixed effects } \\
\text { estimator }\end{array}$ & 1 & 2 & 3 & 4 & 5 & 6 & 7 & 8 & 9 & 10 \\
\hline $\begin{array}{l}\text { Dependent } \\
\text { variables } \\
\text { (in log) }\end{array}$ & Gini & Q1 & Q2 & Q1/Q5 & $(\mathrm{Q} 1+\mathrm{Q} 2) / \mathrm{Q} 5$ & Gini & Q1 & Q2 & Q1/Q5 & $\begin{array}{c}(\mathrm{Q} 1+\mathrm{Q} 2) / \mathrm{Q} \\
5\end{array}$ \\
\hline $\begin{array}{l}\text { GDP per capita } \\
\text { volatility }\end{array}$ & $\begin{array}{c}0.062^{* * *} \\
(0.017)\end{array}$ & $\begin{array}{c}-0.106^{* * *} \\
(0.033)\end{array}$ & $\begin{array}{c}-0.077^{* * *} \\
(0.016)\end{array}$ & $\begin{array}{c}-0.160^{* * *} \\
(0.043)\end{array}$ & $\begin{array}{c}-0.144^{* * *} \\
(0.032)\end{array}$ & $\begin{array}{c}0.084^{* * *} \\
(0.030)\end{array}$ & $\begin{array}{c}-0.183^{* * *} \\
(0.060)\end{array}$ & $\begin{array}{c}-0.125^{* * *} \\
(0.034)\end{array}$ & $\begin{array}{c}-0.275^{* * *} \\
(0.080)\end{array}$ & $\begin{array}{c}-0.241^{* * *} \\
(0.063)\end{array}$ \\
\hline $\begin{array}{l}\text { Ratio of Net } \\
\text { ODA to GDP (in } \\
\text { log) }\end{array}$ & $\begin{array}{l}-0.008 \\
(0.010)\end{array}$ & $\begin{array}{l}-0.035^{*} \\
(0.021)\end{array}$ & $\begin{array}{l}-0.006 \\
(0.011)\end{array}$ & $\begin{array}{c}-0.040+ \\
(0.026)\end{array}$ & $\begin{array}{l}-0.020 \\
(0.020)\end{array}$ & & & & & \\
\hline No ODA dummy & $\begin{array}{l}-0.048 \\
(0.135)\end{array}$ & $\begin{array}{l}-0.381 \\
(0.291)\end{array}$ & $\begin{array}{c}0.012 \\
(0.195)\end{array}$ & $\begin{array}{l}-0.419 \\
(0.371)\end{array}$ & $\begin{array}{l}-0.148 \\
(0.305)\end{array}$ & & & & & \\
\hline $\begin{array}{l}\text { Volatility } x \\
\text { ODA }\end{array}$ & $\begin{array}{l}-0.004 \\
(0.004)\end{array}$ & $\begin{array}{l}0.013^{* *} \\
(0.006)\end{array}$ & $\begin{array}{l}0.005+ \\
(0.003)\end{array}$ & $\begin{array}{l}0.017^{* *} \\
(0.008)\end{array}$ & $\begin{array}{l}0.013^{*} \\
(0.007)\end{array}$ & & & & & \\
\hline $\begin{array}{l}\text { Ratio of } \\
\text { Remittances to } \\
\text { GDP (in log) }\end{array}$ & & & & & & $\begin{array}{l}0.015+ \\
(0.010)\end{array}$ & $\begin{array}{c}-0.061^{* * *} \\
(0.017)\end{array}$ & $\begin{array}{c}-0.033^{* * *} \\
(0.010)\end{array}$ & $\begin{array}{c}-0.081^{* * *} \\
(0.022)\end{array}$ & $\begin{array}{c}-0.062^{* * *} \\
(0.018)\end{array}$ \\
\hline $\begin{array}{l}\text { Volatility x } \\
\text { Remittances }\end{array}$ & & & & & & $\begin{array}{c}-0.024+ \\
(0.016)\end{array}$ & $\begin{array}{l}0.057^{*} \\
(0.029)\end{array}$ & $\begin{array}{l}0.035^{* *} \\
(0.018)\end{array}$ & $\begin{array}{l}0.084^{* *} \\
(0.041)\end{array}$ & $\begin{array}{l}0.072^{* *} \\
(0.033)\end{array}$ \\
\hline $\begin{array}{l}\text { GDP per capita } \\
\text { (in log) }\end{array}$ & $\begin{array}{l}0.512^{*} \\
(0.263)\end{array}$ & $\begin{array}{c}-1.294^{* * *} \\
(0.414)\end{array}$ & $\begin{array}{l}-0.581^{* *} \\
(0.226)\end{array}$ & $\begin{array}{c}-1.738^{* * *} \\
(0.537)\end{array}$ & $\begin{array}{c}-1.242^{* * *} \\
(0.410)\end{array}$ & $\begin{array}{l}0.544^{*} \\
(0.307)\end{array}$ & $\begin{array}{c}-1.217^{* *} \\
(0.525)\end{array}$ & $\begin{array}{c}-0.656^{* * *} \\
(0.250)\end{array}$ & $\begin{array}{c}-1.707^{* * *} \\
(0.652)\end{array}$ & $\begin{array}{c}-1.314^{* * *} \\
(0.472)\end{array}$ \\
\hline $\begin{array}{l}\text { GDP per capita } \\
\text { squared (in log) }\end{array}$ & $\begin{array}{c}-0.032^{* *} \\
(0.015)\end{array}$ & $\begin{array}{c}0.081^{* * *} \\
(0.027)\end{array}$ & $\begin{array}{l}0.034^{* *} \\
(0.014)\end{array}$ & $\begin{array}{c}0.108^{* * *} \\
(0.035)\end{array}$ & $\begin{array}{c}0.076^{* * *} \\
(0.026)\end{array}$ & $\begin{array}{l}-0.034^{*} \\
(0.018)\end{array}$ & $\begin{array}{l}0.075^{* *} \\
(0.036)\end{array}$ & $\begin{array}{l}0.040^{* *} \\
(0.016)\end{array}$ & $\begin{array}{l}0.107^{* *} \\
(0.044)\end{array}$ & $\begin{array}{c}0.082^{* * *} \\
(0.031)\end{array}$ \\
\hline $\begin{array}{l}\text { Population } \\
\text { growth }\end{array}$ & $\begin{array}{l}-0.008 \\
(0.018)\end{array}$ & $\begin{array}{c}0.020 \\
(0.040)\end{array}$ & $\begin{array}{c}0.015 \\
(0.022)\end{array}$ & $\begin{array}{c}0.025 \\
(0.051)\end{array}$ & $\begin{array}{c}0.022 \\
(0.039)\end{array}$ & $\begin{array}{l}-0.020 \\
(0.019)\end{array}$ & $\begin{array}{c}0.017 \\
(0.042)\end{array}$ & $\begin{array}{c}0.012 \\
(0.021)\end{array}$ & $\begin{array}{c}0.020 \\
(0.052)\end{array}$ & $\begin{array}{c}0.017 \\
(0.038)\end{array}$ \\
\hline $\begin{array}{l}\text { Rural } \\
\text { population (in } \\
\text { log) }\end{array}$ & $\begin{array}{l}-0.022 \\
(0.038)\end{array}$ & $\begin{array}{l}0.090^{*} \\
(0.054)\end{array}$ & $\begin{array}{c}0.022 \\
(0.032)\end{array}$ & $\begin{array}{l}0.106+ \\
(0.069)\end{array}$ & $\begin{array}{c}0.059 \\
(0.055)\end{array}$ & $\begin{array}{l}-0.016 \\
(0.076)\end{array}$ & $\begin{array}{c}0.092 \\
(0.126)\end{array}$ & $\begin{array}{c}0.043 \\
(0.052)\end{array}$ & $\begin{array}{c}0.128 \\
(0.145)\end{array}$ & $\begin{array}{c}0.086 \\
(0.098)\end{array}$ \\
\hline Inflation (in log) & $\begin{array}{c}0.012 \\
(0.012)\end{array}$ & $\begin{array}{l}-0.013 \\
(0.023)\end{array}$ & $\begin{array}{c}0.002 \\
(0.010)\end{array}$ & $\begin{array}{l}-0.016 \\
(0.029)\end{array}$ & $\begin{array}{l}-0.006 \\
(0.021)\end{array}$ & $\begin{array}{c}0.008 \\
(0.009)\end{array}$ & $\begin{array}{l}-0.014 \\
(0.022)\end{array}$ & $\begin{array}{c}0.001 \\
(0.009)\end{array}$ & $\begin{array}{l}-0.013 \\
(0.027)\end{array}$ & $\begin{array}{l}-0.002 \\
(0.018)\end{array}$ \\
\hline $\begin{array}{l}\text { Secondary } \\
\text { school } \\
\text { enrolment } \\
\text { (gross, in log) }\end{array}$ & $\begin{array}{c}-0.112^{* *} \\
(0.056)\end{array}$ & $\begin{array}{l}0.265^{* *} \\
(0.102)\end{array}$ & $\begin{array}{c}0.175^{* * *} \\
(0.062)\end{array}$ & $\begin{array}{c}0.372^{* * *} \\
(0.136)\end{array}$ & $\begin{array}{c}0.307^{* * *} \\
(0.109)\end{array}$ & $\begin{array}{c}-0.098+ \\
(0.061)\end{array}$ & $\begin{array}{l}0.275^{* *} \\
(0.110)\end{array}$ & $\begin{array}{c}0.199 * * * \\
(0.069)\end{array}$ & $\begin{array}{c}0.393^{* * *} \\
(0.145)\end{array}$ & $\begin{array}{c}0.338^{* * *} \\
(0.119)\end{array}$ \\
\hline $\begin{array}{l}\text { Ratio of } \\
\text { Government } \\
\text { expenditure to } \\
\text { GDP (in log) }\end{array}$ & $\begin{array}{c}0.069 \\
(0.050)\end{array}$ & $\begin{array}{l}-0.198^{*} \\
(0.100)\end{array}$ & $\begin{array}{c}-0.139 * * * \\
(0.051)\end{array}$ & $\begin{array}{l}-0.250^{*} \\
(0.128)\end{array}$ & $\begin{array}{c}-0.232^{* *} \\
(0.093)\end{array}$ & $\begin{array}{l}0.075+ \\
(0.050)\end{array}$ & $\begin{array}{c}-0.305^{* * *} \\
(0.091)\end{array}$ & $\begin{array}{c}-0.175^{* * *} \\
(0.046)\end{array}$ & $\begin{array}{c}-0.379 * * * \\
(0.116)\end{array}$ & $\begin{array}{c}-0.310 * * * \\
(0.084)\end{array}$ \\
\hline $\begin{array}{l}\text { Consumption } \\
\text { dummy }\end{array}$ & $\begin{array}{l}-0.032 \\
(0.028)\end{array}$ & $\begin{array}{c}0.069 \\
(0.058)\end{array}$ & $\begin{array}{c}0.033 \\
(0.036)\end{array}$ & $\begin{array}{c}0.085 \\
(0.078)\end{array}$ & $\begin{array}{c}0.054 \\
(0.063)\end{array}$ & $\begin{array}{l}-0.024 \\
(0.028)\end{array}$ & $\begin{array}{c}0.072 \\
(0.055)\end{array}$ & $\begin{array}{c}0.031 \\
(0.033)\end{array}$ & $\begin{array}{c}0.092 \\
(0.072)\end{array}$ & $\begin{array}{c}0.058 \\
(0.057)\end{array}$ \\
\hline $\begin{array}{l}\text { Number of } \\
\text { observations }\end{array}$ & 514 & 471 & 469 & 471 & 469 & 470 & 435 & 433 & 435 & 433 \\
\hline $\begin{array}{l}\text { Number of } \\
\text { countries }\end{array}$ & 142 & 140 & 140 & 140 & 140 & 136 & 135 & 135 & 135 & 135 \\
\hline
\end{tabular}

Notes: Robust standard errors in parentheses, $+p<0.15,{ }^{*} p<0.10,{ }^{* *} p<0.05,{ }^{* * *} p<0.01$. Each specification includes period dummies and a constant.

Sources: Authors' calculations based on UNU-WIDER, World Bank and OECD data 
Table 4: Income inequality (Gini and Income share quintiles), volatility, aid and remittances, Sys-GMM, 1973-2012, 5-year periods

\begin{tabular}{|c|c|c|c|c|c|c|c|c|c|c|}
\hline & 1 & 2 & 3 & 4 & 5 & 6 & 7 & 8 & 9 & 10 \\
\hline $\begin{array}{l}\text { Dependent } \\
\text { variables } \\
\text { (in log) }\end{array}$ & Gini & Q1 & Q2 & Q1/Q5 & $(\mathrm{Q} 1+\mathrm{Q} 2) / \mathrm{Q} 5$ & Gini & Q1 & Q2 & Q1/Q5 & $(\mathrm{Q} 1+\mathrm{Q} 2) / \mathrm{Q} 5$ \\
\hline $\begin{array}{l}\text { Lagged } \\
\text { dependent }\end{array}$ & $\begin{array}{c}0.463^{* * *} \\
(4.455)\end{array}$ & $\begin{array}{c}0.339^{* * *} \\
(2.875)\end{array}$ & $\begin{array}{c}0.384^{* * *} \\
(3.049)\end{array}$ & $\begin{array}{l}0.278^{* *} \\
(2.163)\end{array}$ & $\begin{array}{l}0.299 * * \\
(2.376)\end{array}$ & $\begin{array}{c}0.418^{* * * *} \\
(3.434)\end{array}$ & $\begin{array}{c}0.505^{* * *} \\
(4.094)\end{array}$ & $\begin{array}{c}0.464^{* * *} \\
(3.048)\end{array}$ & $\begin{array}{c}0.373^{* * *} \\
(3.356)\end{array}$ & $\begin{array}{c}0.412^{* * * *} \\
(3.743)\end{array}$ \\
\hline $\begin{array}{l}\text { GDP per capita } \\
\text { volatility }\end{array}$ & $\begin{array}{l}0.058^{* *} \\
(2.125)\end{array}$ & $\begin{array}{l}-0.104+ \\
(-1.454)\end{array}$ & $\begin{array}{l}-0.054 \\
(-1.411)\end{array}$ & $\begin{array}{l}-0.203^{* *} \\
(-2.058)\end{array}$ & $\begin{array}{l}-0.114+ \\
(-1.658)\end{array}$ & $\begin{array}{c}0.076 \\
(0.883)\end{array}$ & $\begin{array}{l}-0.119 \\
(-0.564)\end{array}$ & $\begin{array}{l}-0.144+ \\
(-1.480)\end{array}$ & $\begin{array}{l}-0.048 \\
(-0.186)\end{array}$ & $\begin{array}{c}-0.054 \\
(-0.282)\end{array}$ \\
\hline $\begin{array}{l}\text { Ratio of Net ODA } \\
\text { to GDP (in log) } \\
\text { No ODA dummy }\end{array}$ & $\begin{array}{l}0.018 * \\
(1.698) \\
0.205+ \\
(1.585)\end{array}$ & $\begin{array}{c}0.000 \\
(0.007) \\
0.272 \\
(0.848)\end{array}$ & $\begin{array}{c}0.003 \\
(0.212) \\
0.137 \\
(0.717)\end{array}$ & $\begin{array}{c}-0.006 \\
(-0.188) \\
0.260 \\
(0.649)\end{array}$ & $\begin{array}{c}-0.000 \\
(-0.003) \\
0.196 \\
(0.685)\end{array}$ & & & & & \\
\hline Volatility x ODA & $\begin{array}{l}-0.015^{*} \\
(-1.805)\end{array}$ & $\begin{array}{l}0.036 * \\
(1.675)\end{array}$ & $\begin{array}{l}0.018+ \\
(1.526)\end{array}$ & $\begin{array}{l}0.069 * * \\
(2.153)\end{array}$ & $\begin{array}{l}0.039 * \\
(1.757)\end{array}$ & & & & & \\
\hline $\begin{array}{l}\text { Ratio of } \\
\text { Remittances to } \\
\text { GDP (in log) }\end{array}$ & & & & & & $\begin{array}{l}0.003 \\
(0.172)\end{array}$ & $\begin{array}{c}0.016 \\
(0.468)\end{array}$ & $\begin{array}{l}-0.016 \\
(-0.934)\end{array}$ & $\begin{array}{c}0.010 \\
(0.263)\end{array}$ & $\begin{array}{l}0.011 \\
(0.385)\end{array}$ \\
\hline $\begin{array}{l}\text { Volatility } x \\
\text { Remittances }\end{array}$ & & & & & & $\begin{array}{c}0.005 \\
(0.127)\end{array}$ & $\begin{array}{l}-0.078 \\
(-0.765)\end{array}$ & $\begin{array}{c}0.039 \\
(0.838)\end{array}$ & $\begin{array}{l}-0.060 \\
(-0.519)\end{array}$ & $\begin{array}{c}-0.022 \\
(-0.246)\end{array}$ \\
\hline $\begin{array}{l}\text { GDP per capita (in } \\
\text { log) }\end{array}$ & $\begin{array}{c}0.432^{* * *} \\
(4.813)\end{array}$ & $\begin{array}{l}-0.368^{*} \\
(-1.857)\end{array}$ & $\begin{array}{l}-0.278^{* * *} \\
(-2.669)\end{array}$ & $\begin{array}{l}-0.673^{* *} \\
(-2.338)\end{array}$ & $\begin{array}{l}-0.707^{* * *} \\
(-2.999)\end{array}$ & $\begin{array}{c}0.369^{* * *} \\
(3.383)\end{array}$ & $\begin{array}{l}-0.475^{*} \\
(-1.967)\end{array}$ & $\begin{array}{l}-0.316 * * \\
(-2.388)\end{array}$ & $\begin{array}{c}-0.799 * * * \\
(-2.854)\end{array}$ & $\begin{array}{c}-0.683^{* * *} \\
(-3.031)\end{array}$ \\
\hline $\begin{array}{l}\text { GDP per capita } \\
\text { squared (in log) }\end{array}$ & $\begin{array}{c}-0.025^{* * *} \\
(-4.876)\end{array}$ & $\begin{array}{l}0.019+ \\
(1.656)\end{array}$ & $\begin{array}{c}0.016^{* * *} \\
(2.631)\end{array}$ & $\begin{array}{l}0.037^{* *} \\
(2.135)\end{array}$ & $\begin{array}{c}0.041^{* * *} \\
(3.100)\end{array}$ & $\begin{array}{c}-0.022^{* * *} \\
(-3.248)\end{array}$ & $\begin{array}{l}0.027^{* *} \\
(1.989)\end{array}$ & $\begin{array}{l}0.019 * * \\
(2.516)\end{array}$ & $\begin{array}{c}0.047^{* * *} \\
(2.992)\end{array}$ & $\begin{array}{c}0.041^{* * *} \\
(3.145)\end{array}$ \\
\hline Population growth & $\begin{array}{c}0.050^{* * * *} \\
(3.737)\end{array}$ & $\begin{array}{l}-0.052^{*} \\
(-1.798)\end{array}$ & $\begin{array}{l}-0.055^{* *} \\
(-2.584)\end{array}$ & $\begin{array}{c}-0.094^{* * *} \\
(-2.821)\end{array}$ & $\begin{array}{c}-0.093^{* * *} \\
(-3.052)\end{array}$ & $\begin{array}{c}0.049^{* * *} \\
(2.818)\end{array}$ & $\begin{array}{c}-0.044^{* *} \\
(-2.336)\end{array}$ & $\begin{array}{c}-0.045^{* * *} \\
(-2.702)\end{array}$ & $\begin{array}{c}-0.084^{* * *} \\
(-2.646)\end{array}$ & $\begin{array}{c}-0.084^{* * * *} \\
(-3.067)\end{array}$ \\
\hline $\begin{array}{l}\text { Rural population } \\
\text { (in log) }\end{array}$ & $\begin{array}{c}0.008 \\
(0.363)\end{array}$ & $\begin{array}{l}-0.015 \\
(-0.468)\end{array}$ & $\begin{array}{c}0.003 \\
(0.137)\end{array}$ & $\begin{array}{c}-0.011 \\
(-0.277)\end{array}$ & $\begin{array}{l}-0.005 \\
(-0.119)\end{array}$ & $\begin{array}{c}0.012 \\
(0.499)\end{array}$ & $\begin{array}{c}0.020 \\
(0.532)\end{array}$ & $\begin{array}{c}0.007 \\
(0.396)\end{array}$ & $\begin{array}{c}0.005 \\
(0.079)\end{array}$ & $\begin{array}{l}0.002 \\
(0.052)\end{array}$ \\
\hline Inflation (in log) & $\begin{array}{c}0.015 \\
(1.310)\end{array}$ & $\begin{array}{l}-0.029 \\
(-1.034)\end{array}$ & $\begin{array}{l}-0.014 \\
(-1.185)\end{array}$ & $\begin{array}{c}-0.033 \\
(-0.962)\end{array}$ & $\begin{array}{c}-0.020 \\
(-0.789)\end{array}$ & $\begin{array}{c}0.015 \\
(1.349)\end{array}$ & $\begin{array}{l}-0.037+ \\
(-1.486)\end{array}$ & $\begin{array}{c}-0.006 \\
(-0.474)\end{array}$ & $\begin{array}{l}-0.056+ \\
(-1.545)\end{array}$ & $\begin{array}{c}-0.032 \\
(-1.318)\end{array}$ \\
\hline $\begin{array}{l}\text { Secondary school } \\
\text { enrolment (gross, } \\
\text { in log) }\end{array}$ & $\begin{array}{l}-0.004 \\
(-0.090)\end{array}$ & $\begin{array}{c}0.015 \\
(0.211)\end{array}$ & $\begin{array}{c}0.010 \\
(0.248)\end{array}$ & $\begin{array}{c}0.040 \\
(0.425)\end{array}$ & $\begin{array}{c}0.054 \\
(0.645)\end{array}$ & $\begin{array}{c}-0.031 \\
(-0.639)\end{array}$ & $\begin{array}{c}0.030 \\
(0.411)\end{array}$ & $\begin{array}{c}0.009 \\
(0.226)\end{array}$ & $\begin{array}{c}0.077 \\
(0.964)\end{array}$ & $\begin{array}{c}0.043 \\
(0.554)\end{array}$ \\
\hline $\begin{array}{l}\text { Ratio of } \\
\text { Government } \\
\text { expenditure to } \\
\text { GDP (in log) }\end{array}$ & $\begin{array}{c}-0.113^{* * * *} \\
(-3.326)\end{array}$ & $\begin{array}{l}0.087+ \\
(1.535)\end{array}$ & $\begin{array}{c}0.054 \\
(1.351)\end{array}$ & $\begin{array}{l}0.199 * * \\
(2.392)\end{array}$ & $\begin{array}{l}0.123^{* *} \\
(2.018)\end{array}$ & $\begin{array}{c}-0.076^{* * * *} \\
(-2.863)\end{array}$ & $\begin{array}{l}0.134^{* *} \\
(2.470)\end{array}$ & $\begin{array}{l}0.075^{*} \\
(1.852)\end{array}$ & $\begin{array}{c}0.194^{* * * *} \\
(2.736)\end{array}$ & $\begin{array}{c}0.159 * * * \\
(2.660)\end{array}$ \\
\hline $\begin{array}{l}\text { Consumption } \\
\text { dummy }\end{array}$ & $\begin{array}{l}-0.056^{*} \\
(-1.976)\end{array}$ & $\begin{array}{l}0.110^{* *} \\
(2.072)\end{array}$ & $\begin{array}{l}0.052^{*} \\
(1.738)\end{array}$ & $\begin{array}{l}0.130^{* *} \\
(1.991)\end{array}$ & $\begin{array}{l}0.079+ \\
(1.478)\end{array}$ & $\begin{array}{l}-0.062+ \\
(-1.616)\end{array}$ & $\begin{array}{c}0.080 \\
(1.207)\end{array}$ & $\begin{array}{l}0.064+ \\
(1.588)\end{array}$ & $\begin{array}{l}0.146^{*} \\
(1.837)\end{array}$ & $\begin{array}{l}0.112^{*} \\
(1.828)\end{array}$ \\
\hline $\begin{array}{l}\text { Number of } \\
\text { observations }\end{array}$ & 415 & 354 & 351 & 354 & 351 & 387 & 335 & 332 & 335 & 332 \\
\hline $\begin{array}{l}\text { Number of } \\
\text { countries }\end{array}$ & 122 & 116 & 115 & 116 & 115 & 117 & 111 & 110 & 111 & 110 \\
\hline AR1 ( $p$-value) & 0.001 & 0.053 & 0.003 & 0.043 & 0.012 & 0.002 & 0.050 & 0.004 & 0.034 & 0.005 \\
\hline AR2 (p-value) & 0.416 & 0.816 & 0.477 & 0.995 & 0.989 & 0.788 & 0.480 & 0.447 & 0.510 & 0.573 \\
\hline $\begin{array}{l}\text { Hansen test ( } p- \\
\text { value) }\end{array}$ & 0.567 & 0.488 & 0.539 & 0.614 & 0.429 & 0.338 & 0.481 & 0.482 & 0.587 & 0.434 \\
\hline $\begin{array}{l}\text { Number of } \\
\text { instruments }\end{array}$ & 98 & 95 & 95 & 95 & 95 & 81 & 81 & 81 & 81 & 81 \\
\hline
\end{tabular}

Notes: Robust standard errors in parentheses (using the Windmeijer correction), $+p<0.15,{ }^{*} p<0.10,{ }^{* *} p<0.05$, *** $p<0.01$. Each specification includes period dummies and a constant.

Sources: Authors' calculations based on UNU-WIDER, World Bank and OECD data. 
Table 5: Income inequality (Gini and Income share quintiles), remittances and aid, Sys-GMM, 19732012, 5-year periods.

\begin{tabular}{|c|c|c|c|c|c|}
\hline & 1 & 2 & 3 & 4 & 5 \\
\hline \multicolumn{6}{|l|}{ Sys-GMM - Internal instruments } \\
\hline Dependent variables (in log) & Gini & Q1 & Q2 & Q1/Q5 & $(\mathrm{Q} 1+\mathrm{Q} 2) / \mathrm{Q} 5$ \\
\hline \multirow[t]{2}{*}{ Lagged dependent } & $0.550 * * *$ & $0.414^{* * *}$ & $0.542 * * *$ & $0.419 * * *$ & $0.490^{* * *}$ \\
\hline & $(0.098)$ & $(0.114)$ & $(0.133)$ & $(0.116)$ & $(0.107)$ \\
\hline \multirow[t]{2}{*}{ GDP per capita volatility } & 0.079 & -0.067 & -0.070 & 0.000 & 0.004 \\
\hline & $(0.076)$ & $(0.239)$ & $(0.091)$ & $(0.263)$ & $(0.193)$ \\
\hline \multirow[t]{2}{*}{ Ratio of Net ODA to GDP (in log) } & -0.005 & -0.013 & 0.008 & -0.003 & 0.019 \\
\hline & $(0.011)$ & $(0.022)$ & $(0.014)$ & $(0.032)$ & $(0.026)$ \\
\hline \multirow[t]{2}{*}{ No ODA dummy } & 0.009 & 0.026 & 0.117 & 0.136 & 0.259 \\
\hline & $(0.138)$ & $(0.282)$ & $(0.192)$ & $(0.410)$ & $(0.357)$ \\
\hline \multirow[t]{2}{*}{ Volatility $x$ ODA } & -0.006 & $0.056^{*}$ & $0.022+$ & $0.060+$ & 0.031 \\
\hline & $(0.016)$ & $(0.030)$ & $(0.013)$ & $(0.039)$ & $(0.026)$ \\
\hline \multirow[t]{2}{*}{ Ratio of Remittances to GDP (in log) } & -0.013 & 0.028 & 0.001 & 0.027 & 0.011 \\
\hline & $(0.018)$ & $(0.030)$ & $(0.020)$ & $(0.037)$ & $(0.023)$ \\
\hline \multirow{2}{*}{ Volatility $x$ remittances } & 0.044 & -0.039 & 0.022 & -0.005 & 0.025 \\
\hline & $(0.052)$ & $(0.083)$ & $(0.047)$ & $(0.126)$ & $(0.080)$ \\
\hline \multirow[t]{2}{*}{ GDP per capita (in log) } & $0.388^{* * *}$ & $-0.437^{*}$ & $-0.230 * *$ & $-0.698^{* *}$ & $-0.495^{* *}$ \\
\hline & $(0.122)$ & $(0.224)$ & $(0.115)$ & $(0.317)$ & $(0.241)$ \\
\hline \multirow[t]{2}{*}{ GDP per capita squared (in log) } & $-0.024^{* * *}$ & $0.025^{*}$ & $0.015^{* *}$ & $0.042^{* *}$ & $0.032^{* *}$ \\
\hline & $(0.007)$ & $(0.013)$ & $(0.007)$ & $(0.020)$ & $(0.015)$ \\
\hline \multirow[t]{2}{*}{ Population growth } & $0.063^{* * *}$ & $-0.074^{* * *}$ & $-0.058^{* * *}$ & $-0.107^{* * *}$ & $-0.089 * * *$ \\
\hline & $(0.020)$ & $(0.024)$ & $(0.017)$ & $(0.037)$ & $(0.031)$ \\
\hline \multirow[t]{2}{*}{ Rural population (in log) } & 0.008 & 0.005 & 0.009 & 0.013 & 0.017 \\
\hline & $(0.019)$ & $(0.031)$ & $(0.016)$ & $(0.039)$ & $(0.037)$ \\
\hline \multirow[t]{2}{*}{ Inflation (in log) } & 0.012 & -0.037 & -0.008 & -0.050 & -0.031 \\
\hline & $(0.010)$ & $(0.027)$ & $(0.014)$ & $(0.040)$ & $(0.024)$ \\
\hline \multirow[t]{2}{*}{ Secondary school enrolment (gross, in log) } & -0.014 & -0.009 & -0.008 & 0.013 & 0.019 \\
\hline & $(0.040)$ & $(0.054)$ & $(0.042)$ & $(0.080)$ & $(0.073)$ \\
\hline \multirow{4}{*}{$\begin{array}{l}\text { Ratio of Government expenditure to GDP } \\
\text { (in log) } \\
\text { Consumption dummy }\end{array}$} & $-0.065^{* *}$ & $0.122^{* *}$ & 0.040 & $0.175^{*}$ & $0.100^{*}$ \\
\hline & $(0.031)$ & $(0.056)$ & $(0.034)$ & $(0.093)$ & $(0.059)$ \\
\hline & $-0.069^{*}$ & $0.107^{* *}$ & 0.042 & 0.104 & 0.047 \\
\hline & $(0.039)$ & $(0.052)$ & $(0.031)$ & $(0.077)$ & $(0.067)$ \\
\hline Number of observations & 381 & 329 & 326 & 329 & 326 \\
\hline Number of countries & 117 & 111 & 110 & 111 & 110 \\
\hline AR1 (p-value) & 0.000 & 0.045 & 0.001 & 0.027 & 0.002 \\
\hline AR2 (p-value) & 0.974 & 0.523 & 0.273 & 0.531 & 0.463 \\
\hline Hansen test ( $p$-value) & 0.552 & 0.763 & 0.554 & 0.559 & 0.649 \\
\hline Number of instruments & 95 & 95 & 95 & 95 & 95 \\
\hline
\end{tabular}

Notes: Robust standard errors in parentheses (using the Windmeijer correction), $+p<0.15,{ }^{*} p<0.10,{ }^{* *} p<0.05$, *** $p<0.01$. Each specification includes period dummies and a constant.

Sources: Authors' calculations based on UNU-WIDER, World Bank and OECD data. 


\section{Robustness checks}

In the next tables, we present an assessment of the robustness of our core results by using different definitions of income volatility. We also check the validity of our results against alternative or even competing hypotheses.

First, we give estimation results in table 6 using an alternative measure of income volatility. In this table, we use a simpler methodology as proposed in Calderon and Levy Yeyati (2009) by using the 5-year standard deviation of the GDP per capita growth rate. These new results are in line with our previous findings regarding the interaction variable aid $x$ volatility. However, using this methodology the results relative to the direct impact of volatility on income inequality are not significant.

The impact of aid on inequality has already been explored by Chauvet and Mesplé-Somps (2007) and Bjornskov (2010), but the two studies, while adopting similar empirical strategies and data, lead to very different results. Both papers use income shares, by quintiles or deciles, as dependent variables. They also both use an interaction variable of aid $x$ democracy. However, while Chauvet and Mesplé-Somps (2007) find that in democracies aid increases the income share of the middle class, Bjornskov (2010) finds that aid disproportionately benefits the $20 \%$ with the highest income. In Table 7 we assess the robustness of our results to the introduction of a democracy variable from the Polity IV database and its interaction term with aid. This interaction term is not significant, and its introduction does not alter our results on the relationship between inequality, aid, and volatility. 
Table 6: Income inequality (Gini and Income share quintiles), alternative measure of volatility (the 5year standard deviation of the GDP per capita growth rate), Sys-GMM, 1973-2012, 5-year periods.

\begin{tabular}{|c|c|c|c|c|c|}
\hline & 1 & 2 & 3 & 4 & 5 \\
\hline \multicolumn{6}{|l|}{ Sys-GMM estimator - Internal instruments } \\
\hline Dependent variables (in log) & Gini & Q1 & Q2 & Q1/Q5 & $(\mathrm{Q} 1+\mathrm{Q} 2) / \mathrm{Q} 5$ \\
\hline \multirow[t]{2}{*}{ Lagged dependent } & $0.717^{* *}$ & $0.318^{* *}$ & $0.439^{* *}$ & $0.461^{* *}$ & $0.495^{* * *}$ \\
\hline & $(0.327)$ & $(0.159)$ & $(0.203)$ & $(0.201)$ & $(0.185)$ \\
\hline \multirow[t]{2}{*}{ GDP per capita growth volatility } & 0.000 & 0.011 & 0.000 & 0.026 & 0.007 \\
\hline & $(0.013)$ & $(0.032)$ & $(0.019)$ & $(0.037)$ & $(0.030)$ \\
\hline \multirow[t]{2}{*}{ Ratio of Net ODA to GDP (in log) } & -0.030 & -0.022 & -0.003 & -0.008 & 0.005 \\
\hline & $(0.023)$ & $(0.045)$ & $(0.027)$ & $(0.054)$ & $(0.044)$ \\
\hline \multirow{2}{*}{ No ODA dummy } & -0.314 & -0.043 & 0.150 & 0.190 & 0.385 \\
\hline & $(0.287)$ & $(0.492)$ & $(0.283)$ & $(0.600)$ & $(0.490)$ \\
\hline \multirow[t]{2}{*}{ Volatility x ODA } & 0.001 & $0.008^{* *}$ & $0.005^{*}$ & $0.009 * *$ & $0.008^{* *}$ \\
\hline & $(0.003)$ & $(0.004)$ & $(0.002)$ & $(0.004)$ & $(0.004)$ \\
\hline \multirow[t]{2}{*}{ GDP per capita (in log) } & 0.171 & $-0.744^{* *}$ & -0.222 & -0.735 & -0.375 \\
\hline & $(0.247)$ & $(0.323)$ & $(0.217)$ & $(0.537)$ & $(0.411)$ \\
\hline \multirow[t]{2}{*}{ GDP per capita squared (in log) } & -0.012 & $0.043^{* *}$ & 0.013 & 0.044 & 0.022 \\
\hline & $(0.016)$ & $(0.020)$ & $(0.013)$ & $(0.035)$ & $(0.026)$ \\
\hline \multirow[t]{2}{*}{ Population growth } & 0.016 & $-0.097^{* *}$ & -0.039 & $-0.103+$ & $-0.072+$ \\
\hline & $(0.037)$ & $(0.039)$ & $(0.028)$ & $(0.065)$ & $(0.049)$ \\
\hline \multirow[t]{2}{*}{ Rural population (in log) } & 0.004 & 0.017 & -0.005 & 0.021 & -0.001 \\
\hline & $(0.012)$ & $(0.035)$ & $(0.020)$ & $(0.043)$ & $(0.030)$ \\
\hline \multirow[t]{2}{*}{ Inflation (in log) } & 0.014 & -0.034 & -0.012 & -0.057 & -0.031 \\
\hline & $(0.015)$ & $(0.037)$ & $(0.019)$ & $(0.049)$ & $(0.035)$ \\
\hline \multirow[t]{2}{*}{ Secondary school enrollment (gross, in log) } & -0.041 & $0.090+$ & 0.047 & 0.103 & 0.076 \\
\hline & $(0.033)$ & $(0.059)$ & $(0.037)$ & $(0.076)$ & $(0.057)$ \\
\hline \multirow{2}{*}{$\begin{array}{l}\text { Ratio of Government expenditure to GDP (in } \\
\text { log) }\end{array}$} & -0.014 & 0.068 & 0.025 & 0.062 & 0.036 \\
\hline & $(0.043)$ & $(0.069)$ & $(0.045)$ & $(0.095)$ & $(0.075)$ \\
\hline \multirow[t]{2}{*}{ Consumption dummy } & $-0.057+$ & 0.076 & 0.049 & 0.088 & 0.063 \\
\hline & $(0.036)$ & $(0.063)$ & $(0.049)$ & $(0.106)$ & $(0.087)$ \\
\hline Number of observations & 466 & 393 & 389 & 392 & 389 \\
\hline Number of countries & 123 & 116 & 115 & 116 & 115 \\
\hline AR1 (p-value) & 0.029 & 0.033 & 0.010 & 0.046 & 0.014 \\
\hline AR2 (p-value) & 0.565 & 0.737 & 0.461 & 0.761 & 0.838 \\
\hline Hansen test ( $p$-value) & 0.849 & 0.826 & 0.340 & 0.741 & 0.797 \\
\hline Number of instruments & 32 & 32 & 32 & 32 & 32 \\
\hline
\end{tabular}

Notes: Robust standard errors in parentheses (using the Windmeijer correction), $+p<0.15,{ }^{*} p<0.10$, ${ }^{* *} p<0.05$, *** $p<0.01$. Each specification includes period dummies and a constant.

Sources: Authors' calculations based on UNU-WIDER, World Bank and OECD data. 
Table 7: Income inequality (Gini and Income share quintiles), volatility, democracy and aid, Sys-GMM, 1973-2012, 5-year periods.

\begin{tabular}{|c|c|c|c|c|c|}
\hline & 1 & 2 & 3 & 4 & 5 \\
\hline \multicolumn{6}{|l|}{ Sys-GMM - Internal instruments } \\
\hline Dependent variables (in log) & Gini & Q1 & Q2 & Q1/Q5 & $(\mathrm{Q} 1+\mathrm{Q} 2) / \mathrm{Q} 5$ \\
\hline Lagged dependent & $\begin{array}{c}0.448^{* * *} \\
(0.105) \\
\end{array}$ & $\begin{array}{c}0.400^{* * *} \\
(0.125) \\
\end{array}$ & $\begin{array}{c}0.517^{* * *} \\
(0.113) \\
\end{array}$ & $\begin{array}{c}0.361^{* * *} \\
(0.107) \\
\end{array}$ & $\begin{array}{c}0.446^{* * *} \\
(0.124)\end{array}$ \\
\hline GDP per capita volatility & $\begin{array}{c}0.080^{* * *} \\
(0.025)\end{array}$ & $\begin{array}{c}-0.034 \\
(0.051)\end{array}$ & $\begin{array}{c}-0.044+ \\
(0.027)\end{array}$ & $\begin{array}{c}-0.033 \\
(0.067)\end{array}$ & $\begin{array}{l}-0.055 \\
(0.053)\end{array}$ \\
\hline Ratio of Net ODA to GDP (in log) & $\begin{array}{c}0.007 \\
(0.010)\end{array}$ & $\begin{array}{l}-0.005 \\
(0.019)\end{array}$ & $\begin{array}{c}0.003 \\
(0.016)\end{array}$ & $\begin{array}{c}0.004 \\
(0.021)\end{array}$ & $\begin{array}{c}0.006 \\
(0.023)\end{array}$ \\
\hline No ODA dummy & $\begin{array}{l}-0.057 \\
(0.120)\end{array}$ & $\begin{array}{l}0.406+ \\
(0.291)\end{array}$ & $\begin{array}{c}0.210 \\
(0.202)\end{array}$ & $\begin{array}{l}0.661^{*} \\
(0.334)\end{array}$ & $\begin{array}{c}0.420 \\
(0.308)\end{array}$ \\
\hline Volatility x ODA & $\begin{array}{c}-0.019 * * \\
(0.008)\end{array}$ & $\begin{array}{c}0.016 \\
(0.015)\end{array}$ & $\begin{array}{l}0.015^{* *} \\
(0.007)\end{array}$ & $\begin{array}{c}0.018 \\
(0.019)\end{array}$ & $\begin{array}{l}0.023+ \\
(0.015)\end{array}$ \\
\hline Polity IV index & $\begin{array}{l}0.006^{* *} \\
(0.003)\end{array}$ & $\begin{array}{l}-0.002 \\
(0.009)\end{array}$ & $\begin{array}{l}-0.002 \\
(0.004)\end{array}$ & $\begin{array}{l}-0.007 \\
(0.009)\end{array}$ & $\begin{array}{l}-0.006 \\
(0.006)\end{array}$ \\
\hline Polity $x$ ODA & $\begin{array}{l}-0.001 \\
(0.001)\end{array}$ & $\begin{array}{l}0.003+ \\
(0.002)\end{array}$ & $\begin{array}{c}0.001 \\
(0.001)\end{array}$ & $\begin{array}{l}0.003+ \\
(0.002)\end{array}$ & $\begin{array}{c}0.002 \\
(0.001)\end{array}$ \\
\hline GDP per capita (in log) & $\begin{array}{c}0.339 * * * \\
(0.101)\end{array}$ & $\begin{array}{c}-0.444^{* *} \\
(0.184)\end{array}$ & $\begin{array}{l}-0.185^{*} \\
(0.100)\end{array}$ & $\begin{array}{c}-0.715^{* *} \\
(0.278)\end{array}$ & $\begin{array}{c}-0.540^{* *} \\
(0.241)\end{array}$ \\
\hline GDP per capita squared (in log) & $\begin{array}{c}-0.021^{* * *} \\
(0.006)\end{array}$ & $\begin{array}{c}0.026^{* *} \\
(0.011)\end{array}$ & $\begin{array}{l}0.011^{*} \\
(0.006)\end{array}$ & $\begin{array}{c}0.043^{* *} \\
(0.016)\end{array}$ & $\begin{array}{l}0.033^{* *} \\
(0.014)\end{array}$ \\
\hline Population growth & $\begin{array}{c}0.051^{* * * *} \\
(0.015)\end{array}$ & $\begin{array}{l}-0.041 \\
(0.031)\end{array}$ & $\begin{array}{c}-0.040^{* * *} \\
(0.016)\end{array}$ & $\begin{array}{l}-0.081^{*} \\
(0.041)\end{array}$ & $\begin{array}{c}-0.078^{* *} \\
(0.035)\end{array}$ \\
\hline Rural population (in log) & $\begin{array}{l}-0.002 \\
(0.020)\end{array}$ & $\begin{array}{c}0.020 \\
(0.042)\end{array}$ & $\begin{array}{c}0.009 \\
(0.015)\end{array}$ & $\begin{array}{c}0.024 \\
(0.045)\end{array}$ & $\begin{array}{c}0.014 \\
(0.041)\end{array}$ \\
\hline Inflation (in log) & $\begin{array}{c}0.012 \\
(0.010)\end{array}$ & $\begin{array}{c}-0.041+ \\
(0.026)\end{array}$ & $\begin{array}{l}-0.011 \\
(0.011)\end{array}$ & $\begin{array}{c}-0.052+ \\
(0.035)\end{array}$ & $\begin{array}{l}-0.028 \\
(0.022)\end{array}$ \\
\hline $\begin{array}{l}\text { Secondary school enrolment (gross, in } \\
\text { log) }\end{array}$ & $\begin{array}{c}0.012 \\
(0.040)\end{array}$ & $\begin{array}{c}0.057 \\
(0.079)\end{array}$ & $\begin{array}{c}0.009 \\
(0.039)\end{array}$ & $\begin{array}{c}0.086 \\
(0.089)\end{array}$ & $\begin{array}{c}0.065 \\
(0.072)\end{array}$ \\
\hline $\begin{array}{l}\text { Ratio of Government expenditure to } \\
\text { GDP (in log) }\end{array}$ & $\begin{array}{c}-0.079^{* *} \\
(0.034)\end{array}$ & $\begin{array}{c}0.086 \\
(0.068)\end{array}$ & $\begin{array}{c}0.023 \\
(0.036)\end{array}$ & $\begin{array}{c}0.127 \\
(0.091)\end{array}$ & $\begin{array}{l}0.073 \\
(0.086)\end{array}$ \\
\hline Consumption dummy & $\begin{array}{l}-0.049^{*} \\
(0.029)\end{array}$ & $\begin{array}{l}0.105+ \\
(0.069)\end{array}$ & $\begin{array}{c}0.044 \\
(0.037)\end{array}$ & $\begin{array}{c}0.127 \\
(0.083)\end{array}$ & $\begin{array}{c}0.077 \\
(0.074)\end{array}$ \\
\hline Number of observations & 401 & 343 & 340 & 343 & 340 \\
\hline Number of countries & 114 & 109 & 108 & 109 & 108 \\
\hline AR1 (p-value) & 0.001 & 0.064 & 0.001 & 0.032 & 0.007 \\
\hline AR2 (p-value) & 0.382 & 0.669 & 0.365 & 0.799 & 0.740 \\
\hline Hansen test ( $p$-value) & 0.280 & 0.280 & 0.354 & 0.352 & 0.229 \\
\hline Number of instruments & 99 & 98 & 98 & 98 & 98 \\
\hline
\end{tabular}

Notes: Robust standard errors in parentheses (using the Windmeijer correction), $+p<0.15,{ }^{*} p<0.10,{ }^{* *} p<0.05$, *** $p<0.01$. Each specification includes period dummies and a constant.

Sources: Authors' calculations based on UNU-WIDER, World Bank and OECD data. 


\section{Discussion-transmission channels of aid and remittances on inequality}

\subsection{Human capital investment as a channel for the mitigating effect of aid}

In line with the literature, our results indicate that output volatility has an adverse effect on income distribution and poverty (channel 1 of Figure 1). We also find that aid tends to dampen this adverse effect (channel 2), while remittances do not ${ }^{6}$. The remaining question relates to the mechanisms that may be at work and which may explain why aid mitigates the negative effect of output volatility on inequality.

One way volatility affects income distribution is by impacting the poorest and richest households in an asymmetric way. The income of the poor may decrease by more during a period of recession than it increases during a period of growth, especially in the absence of adequate social safety nets. This is because the least educated workers are the first to be made redundant and remain unemployed for longer, which makes it less easy for them to find employment when the situation is reversed (Agenor, 2002). Their income, which is generally not indexed to the price of goods, is especially affected in real terms by variations in inflation (inflation not being anticipated) that accompanies financial instability (Guillaumont Jeanneney and Kpodar, 2011). Moreover, output contractions tend to disproportionately affect the poorest households (Calderon and Levy Yeyati, 2009). It is more difficult for the poorest households to cope with adverse income shocks. Their sources of income are less diversified than those of the richest households, and they have little access to credit. In times of output reductions, the poorest people are therefore more likely to cut their investments in physical and human capital. This in turn has long term effects on income distribution and poverty which are difficult to reverse in times of expansion.

One way of getting some insight into this disinvestment channel is first, to simply look at the correlation between education enrolment rates and output volatility. Figure 2 shows that high output volatility is associated with lower education outcomes, ${ }^{7}$ the direction of the causality being unclear. However, foreign aid seems to mitigate this effect. In Figure 3, we plot the same relationship, but here we divide our sample according to the median level of aid in our sample (around 5\% of GDP). Clearly, a negative relationship between output volatility and education is seen for the sample of countries which receive small amounts of aid (grey line), while the fit is flat in the case of the sub-sample of countries which receive larger amounts of aid (black line). This pattern also appears when we divide the sample of countries according to the median value of aid to the social sectors (around 1\% of GDP) (Figure 4). ${ }^{8}$

\footnotetext{
${ }^{6}$ Channel 3 (impact of aid on inequality) is not significant.

${ }^{7}$ The same pattern appears when education is removed from the effect of income per capita.

${ }^{8}$ Aid to social sectors is from the Creditor Reporting System (CRS) dataset and includes aid to education, for health of population, and water and sanitation. It is only available for 2002 onwards.
} 
Figure 2. Enrolment rate and income volatility, 1973-2012, 5-year averages

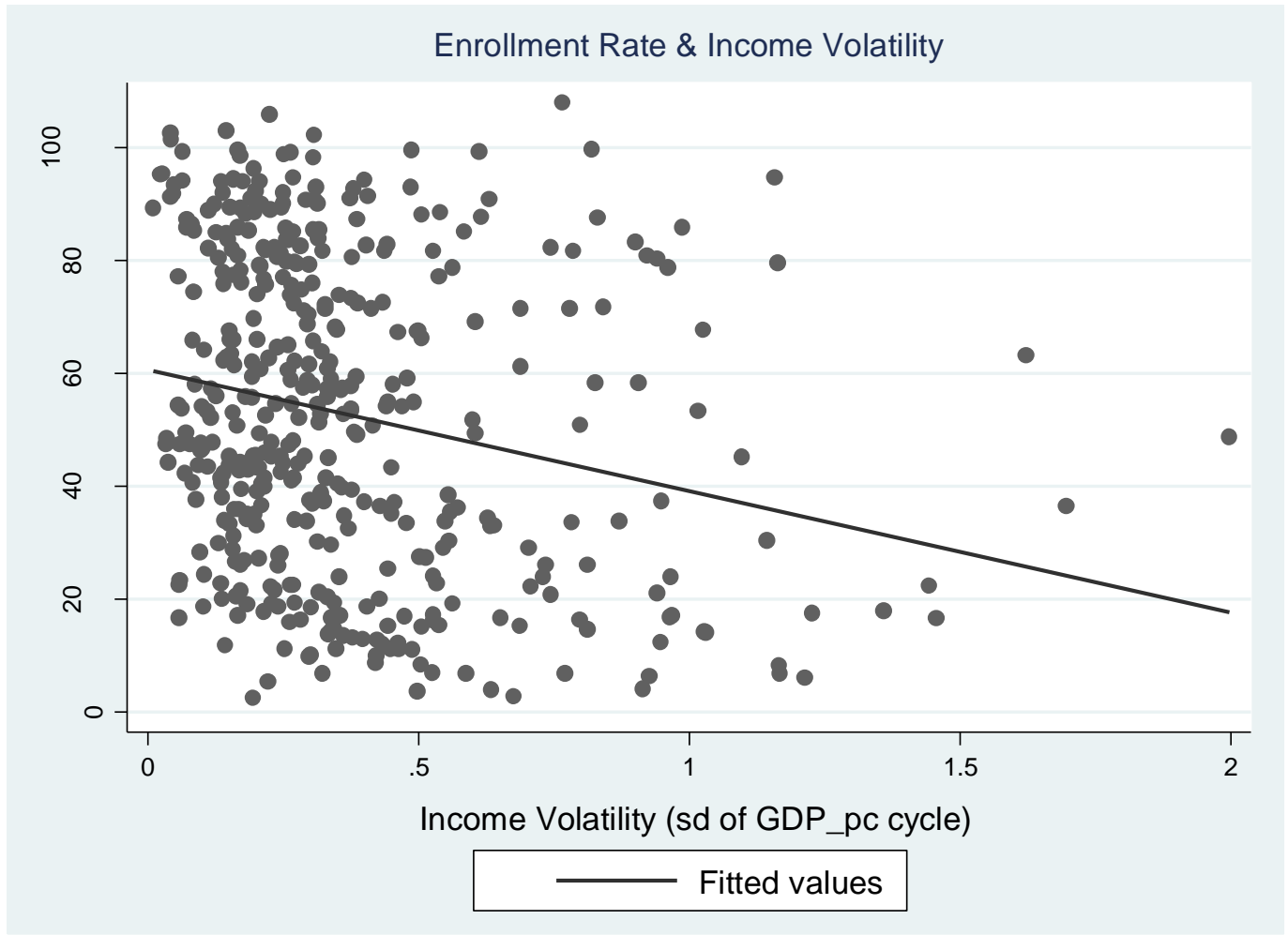

Figure 3. Enrolment rate and income volatility, by levels of aid, 1973-2012, 5-year averages.

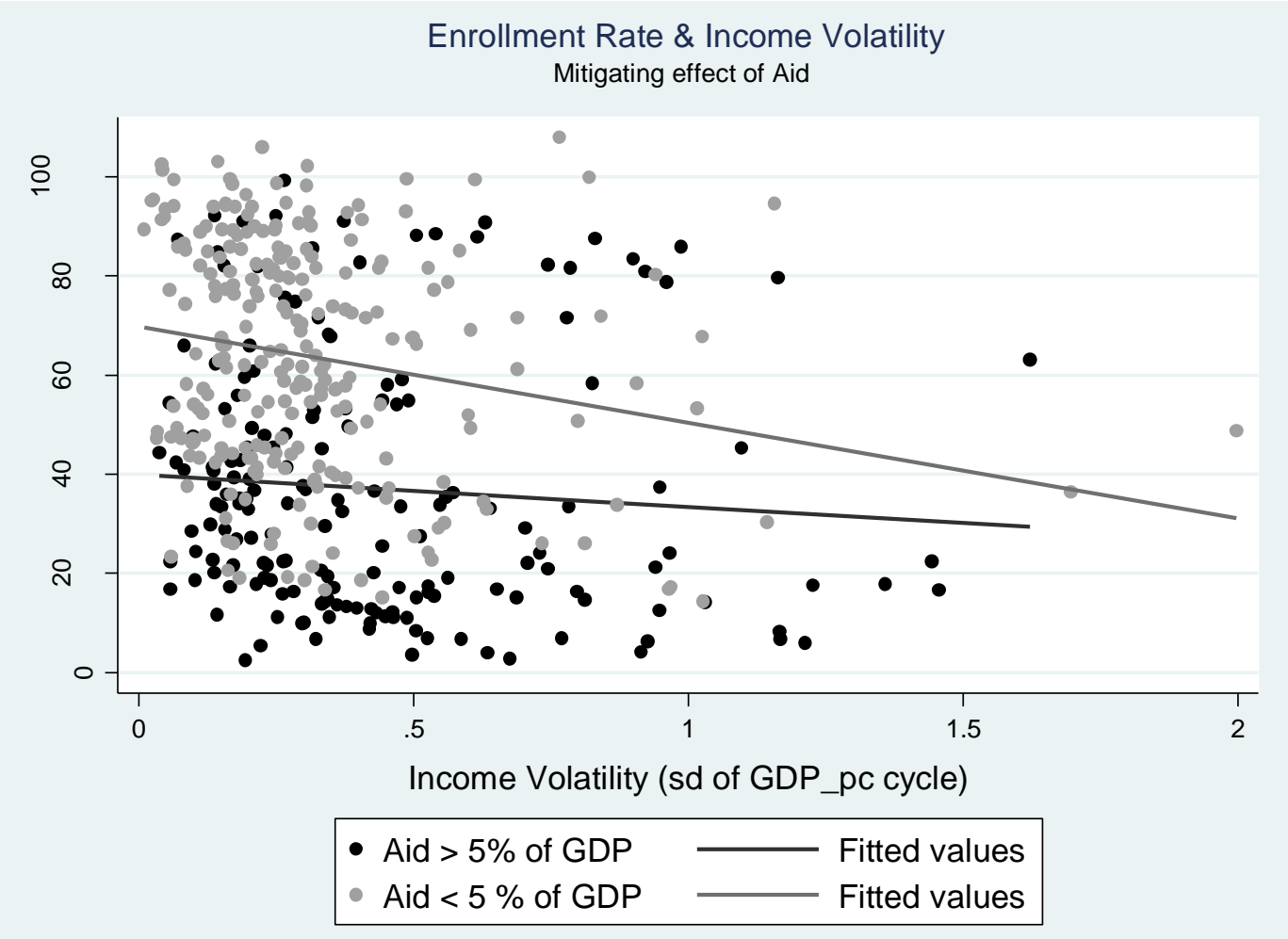


Figure 4. Enrolment rate and income volatility, by levels of aid to the social sectors, 1998-2012, five-year averages

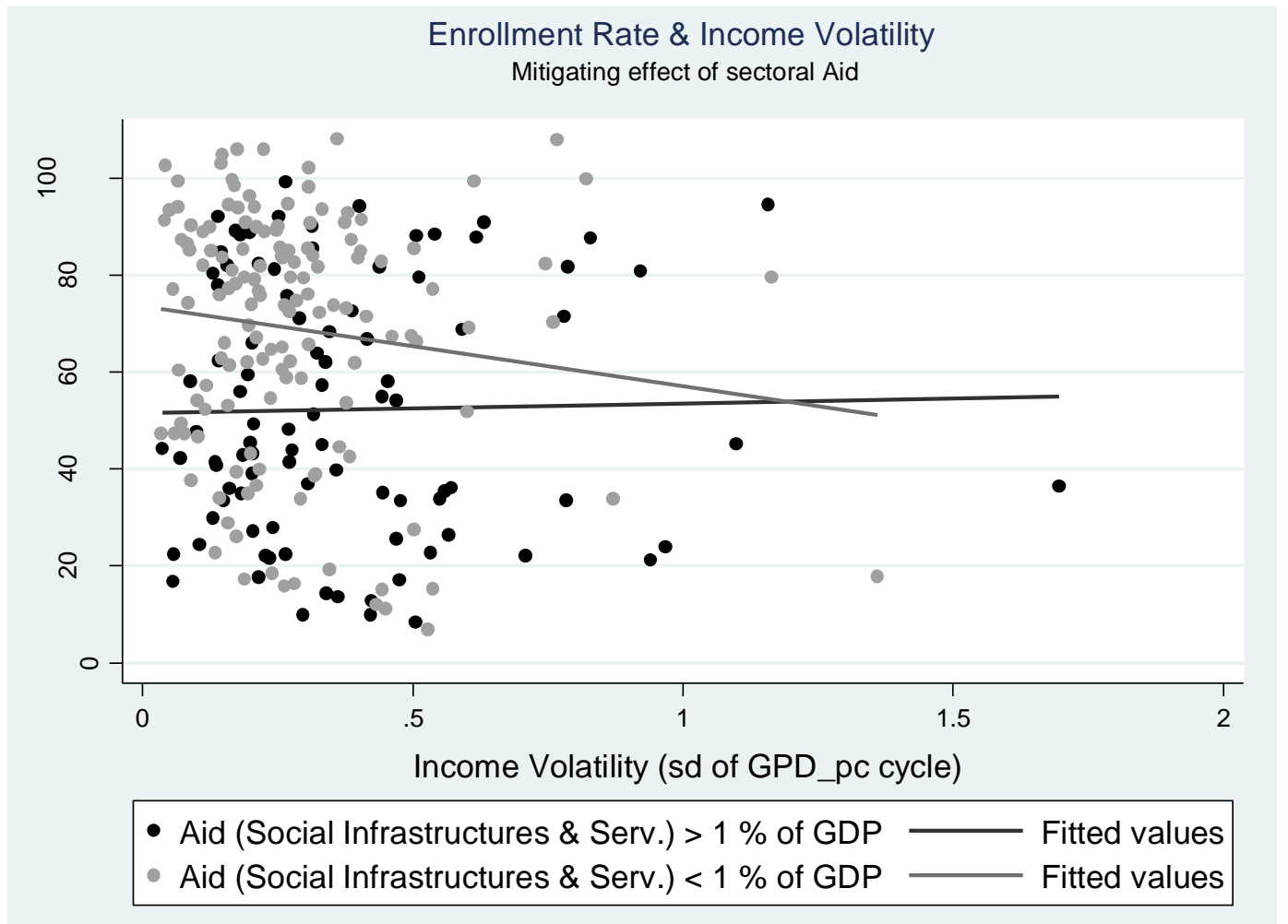

Note: Observations for period 1998-2002 are dropped for aid recipients with no data on aid to social sectors.

These intuitions are supported by the results of Table 8 which shows the effect of income volatility on secondary school enrolment rates as well as the mitigating effect of foreign aid. We use the same empirical strategy as before, and we just change inequality for the secondary school enrolment rate as the dependent variable. We first observe that income volatility has a detrimental effect on school attendance (column 1). This result suggests that one coping strategy of the poor when they face a negative income shock is a cut in their investment in human capital ${ }^{9}$. Next, given our intuitions from Figure 3 and 4, we add to the specification foreign aid as well as an interaction term foreign aid $\mathrm{x}$ income volatility which represents its potential mitigating effect. We find that while aid does not seem to directly affect the secondary school enrolment rate, its interaction with volatility is significant and has the expected sign. According to the results in columns $2 \& 3$, foreign aid tends to dampen the negative impact of income volatility on secondary school attendance, highlighting one potential channel through which the stabilizing effect of aid impacts income inequality. We observe that this effect is robust to the inclusion of remittances, but remittances are

\footnotetext{
${ }^{9}$ Note that when looking at the primary school enrolment rate the effect of macroeconomic volatility is also negative and statistically significant. Results not reported here to save space but available on request from the authors.
} 
not significant. ${ }^{1011}$ Finally, no significant result is observed for remittances, either when they are added to the specification that includes foreign aid, or when aid and remittances are included in a separate equation (not shown). According to these results, it seems that aid is likely to reduce the positive impact of income volatility on inequality in as far as it allows more public spending in favour of the poor (as safety nets or social expenditure). This can in turn prevent school drop-out for the poorest and secure their investment in human capital which, to a larger extent, contributes to decreasing income inequality in the long run.

\subsection{Exploring the direct stabilizing effect of foreign aid and remittances on income volatility}

It may also be the case that aid or remittances reduce the negative effect of income volatility on the poorest segment of the population by decreasing income volatility directly (channel 4 of Figure 1). Developing countries' volatility results both from internal and external factors (Raddatz, 2007). The compensating effect of aid with respect to the external sources of volatility is easier to assess than with respect to the internal ones, given that the former are more likely to be exogenous to aid and to the economic conditions prevailing in the recipient country. In what follows, we provide evidence which suggests that aid tends to dampen the adverse effect of income volatility by stabilizing the flow of external resources. Figure 5 shows the slightly positive relationship between inequality and export volatility (measured in the same way as income volatility, see Equation (2) in Section 2). However, Figure 6 shows that for the sub-sample of countries in which aid is countercyclical with respect to exports, ${ }^{12}$ export volatility is no longer associated with higher inequality (black line). On the contrary, when aid is pro-cyclical, export volatility is associated with higher inequality (grey line).

\footnotetext{
${ }^{10}$ Estimations with remittances only (i.e. without aid) are not shown here in order to save space. They show no significant effect of remittances on secondary school attendance, both in number and in interaction with macroeconomic volatility.

${ }^{11}$ When using the primary instead of the secondary school enrolment rate, the interaction term between aid and macroeconomic volatility is still positive, but less significant (results not shown).

${ }^{12}$ Aid counter-cyclicality is measured using the correlation of the cycles of aid with the cycles of exports. When the correlation is negative, aid is assumed to be counter-cyclical. Aid and exports are measured in constant US dollars deflated by US unit import prices.
} 
Table 8: Secondary school enrolment, income volatility, aid and remittances, Sys-GMM, 1973-2012, 5year periods.

\begin{tabular}{|c|c|c|c|c|c|}
\hline & 1 & 2 & 3 & 4 & 5 \\
\hline $\begin{array}{l}\text { Dependent variables } \\
\text { (school enrolment rate) }\end{array}$ & $\begin{array}{l}\text { secondary } \\
\text { gross }\end{array}$ & $\begin{array}{l}\text { secondary } \\
\text { gross }\end{array}$ & $\begin{array}{l}\text { secondary } \\
\text { net }\end{array}$ & $\begin{array}{l}\text { secondary } \\
\text { gross }\end{array}$ & $\begin{array}{c}\text { secondary } \\
\text { net }\end{array}$ \\
\hline Lagged dependent & $\begin{array}{l}0.881^{* * *} \\
(0.073)\end{array}$ & $\begin{array}{l}0.824^{* * *} \\
(0.029)\end{array}$ & $\begin{array}{l}0.870^{* * *} \\
(0.093)\end{array}$ & $\begin{array}{l}0.769^{* * *} \\
(0.081)\end{array}$ & $\begin{array}{c}0.749 * * * \\
(0.096)\end{array}$ \\
\hline GDP per capita volatility & $\begin{array}{l}-0.143 * * \\
(0.059)\end{array}$ & $\begin{array}{c}-0.046 * * * \\
(0.016)\end{array}$ & $\begin{array}{l}-0.029 \\
(0.079)\end{array}$ & $\begin{array}{l}-0.018 \\
(0.102)\end{array}$ & $\begin{array}{c}0.127 \\
(0.227)\end{array}$ \\
\hline Ratio of Net ODA to GDP (in log) & & $\begin{array}{l}-0.018 \\
(0.015)\end{array}$ & $\begin{array}{c}0.010 \\
(0.028)\end{array}$ & $\begin{array}{l}-0.018 \\
(0.037)\end{array}$ & $\begin{array}{l}-0.003 \\
(0.035)\end{array}$ \\
\hline No ODA dummy & & $\begin{array}{l}-0.235 \\
(0.198)\end{array}$ & $\begin{array}{c}0.210 \\
(0.391)\end{array}$ & $\begin{array}{l}-0.094 \\
(0.550)\end{array}$ & $\begin{array}{c}0.005 \\
(0.472)\end{array}$ \\
\hline Volatility x ODA & & $\begin{array}{l}0.008^{* *} \\
(0.004)\end{array}$ & $\begin{array}{c}0.013^{* * *} \\
(0.004)\end{array}$ & $\begin{array}{c}0.024 \\
(0.021)\end{array}$ & $\begin{array}{c}0.009 \\
(0.012)\end{array}$ \\
\hline Ratio of Remittances to GDP (in log) & & & & $\begin{array}{c}0.026 \\
(0.037)\end{array}$ & $\begin{array}{c}0.019 \\
(0.040)\end{array}$ \\
\hline Volatility x Remittances & & & & $\begin{array}{l}-0.027 \\
(0.073)\end{array}$ & $\begin{array}{l}-0.039 \\
(0.077)\end{array}$ \\
\hline GDP per capita (in log) & $\begin{array}{c}0.046 \\
(0.101)\end{array}$ & $\begin{array}{c}0.107 \\
(0.082)\end{array}$ & $\begin{array}{c}0.263 \\
(0.244)\end{array}$ & $\begin{array}{c}0.240 \\
(0.255)\end{array}$ & $\begin{array}{c}0.234 \\
(0.195)\end{array}$ \\
\hline GDP per capita squared (in log) & $\begin{array}{l}-0.003 \\
(0.005)\end{array}$ & $\begin{array}{l}-0.006 \\
(0.005)\end{array}$ & $\begin{array}{l}-0.016 \\
(0.013)\end{array}$ & $\begin{array}{l}-0.014 \\
(0.015)\end{array}$ & $\begin{array}{l}-0.012 \\
(0.011)\end{array}$ \\
\hline Population growth & $\begin{array}{c}0.004 \\
(0.010)\end{array}$ & $\begin{array}{l}-0.005 \\
(0.007)\end{array}$ & $\begin{array}{c}0.009 \\
(0.010)\end{array}$ & $\begin{array}{c}0.006 \\
(0.017)\end{array}$ & $\begin{array}{l}-0.002 \\
(0.015)\end{array}$ \\
\hline Rural population (in log) & $\begin{array}{c}0.003 \\
(0.014)\end{array}$ & $\begin{array}{l}-0.001 \\
(0.009)\end{array}$ & $\begin{array}{l}-0.004 \\
(0.025)\end{array}$ & $\begin{array}{c}0.005 \\
(0.024)\end{array}$ & $\begin{array}{c}0.002 \\
(0.020)\end{array}$ \\
\hline Inflation (in log) & $\begin{array}{c}0.000 \\
(0.007)\end{array}$ & $\begin{array}{l}-0.007 \\
(0.007)\end{array}$ & $\begin{array}{l}-0.009 \\
(0.011)\end{array}$ & $\begin{array}{l}-0.004 \\
(0.012)\end{array}$ & $\begin{array}{l}-0.008 \\
(0.015)\end{array}$ \\
\hline $\begin{array}{l}\text { Ratio of Government expenditure to } \\
\text { GDP (in log) }\end{array}$ & $\begin{array}{c}0.031 \\
(0.020)\end{array}$ & $\begin{array}{c}0.029 \\
(0.026)\end{array}$ & $\begin{array}{c}0.003 \\
(0.060)\end{array}$ & $\begin{array}{c}0.021 \\
(0.054)\end{array}$ & $\begin{array}{l}-0.006 \\
(0.075)\end{array}$ \\
\hline Consumption dummy & $\begin{array}{c}0.011 \\
(0.023)\end{array}$ & $\begin{array}{l}-0.011 \\
(0.020)\end{array}$ & $\begin{array}{l}-0.017 \\
(0.059)\end{array}$ & $\begin{array}{c}-0.032 \\
(0.028)\end{array}$ & $\begin{array}{c}-0.029 \\
(0.122)\end{array}$ \\
\hline Number of observations & 690 & 684 & 281 & 597 & 245 \\
\hline Number of countries & 162 & 162 & 107 & 152 & 97 \\
\hline AR 1 (p-value) & 0.001 & 0.000 & 0.094 & 0.004 & 0.117 \\
\hline AR 2 (p-avlue) & 0.095 & 0.145 & 0.506 & 0.247 & 0.790 \\
\hline Hansen test ( $p$-value) & 0.326 & 0.622 & 0.802 & 0.225 & 0.207 \\
\hline Number of instruments & 61 & 159 & 51 & 65 & 65 \\
\hline
\end{tabular}

Notes: Robust standard errors in parentheses (using the Windmeijer correction) $+p<0.15,{ }^{*} p<0.10$,

${ }^{* *} p<0.05,{ }^{* * *} p<0.01$. Each specification includes period dummies and a constant.

Sources: Authors' calculations based on UNU-WIDER, World Bank and OECD data. 
Figure 5. Inequality and export volatility, 1973-2012, five-year averages

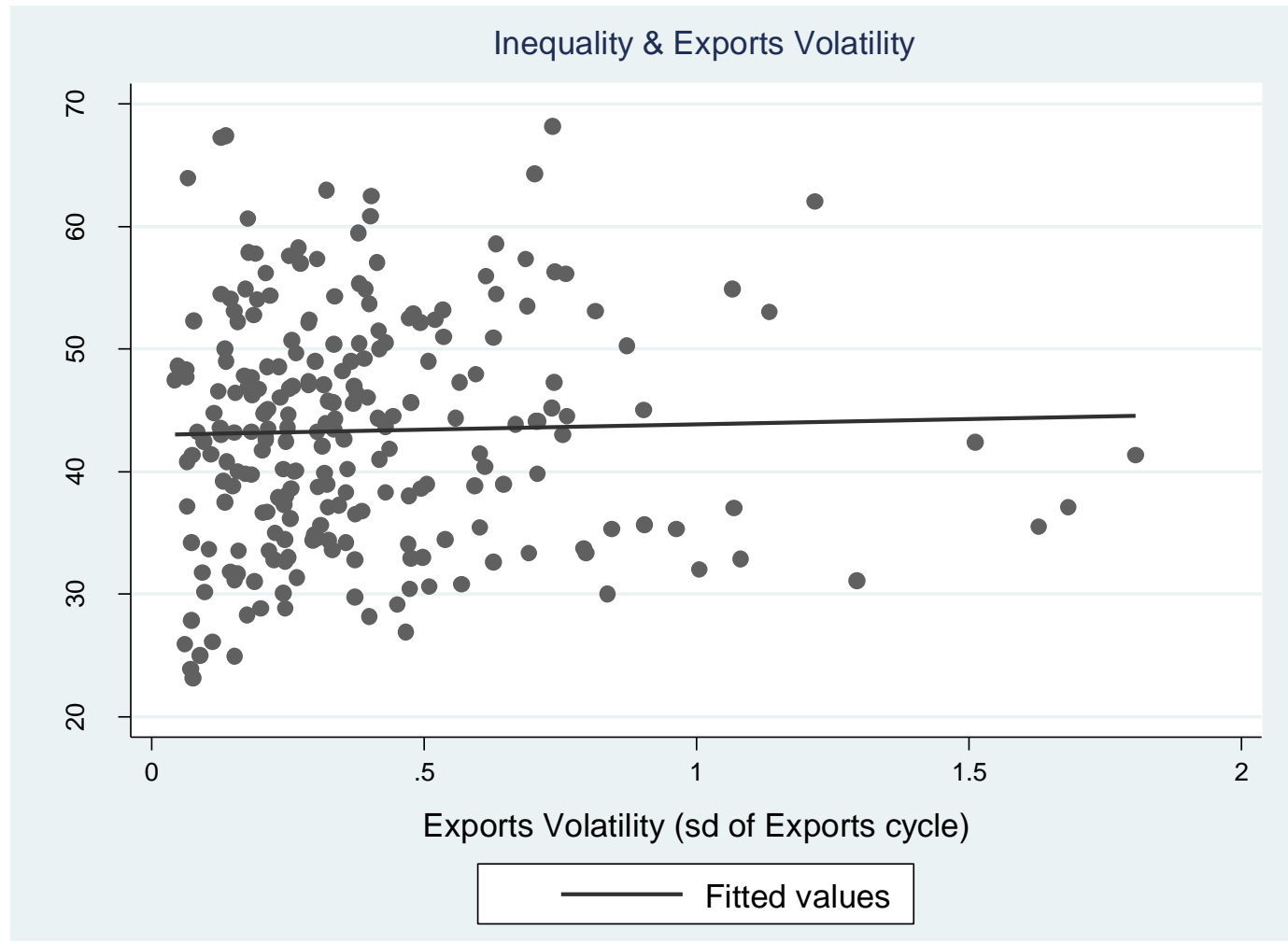

Figure 6. Inequality and exports volatility, depending on counter-cyclicality of aid, 1973-2012, five-year averages

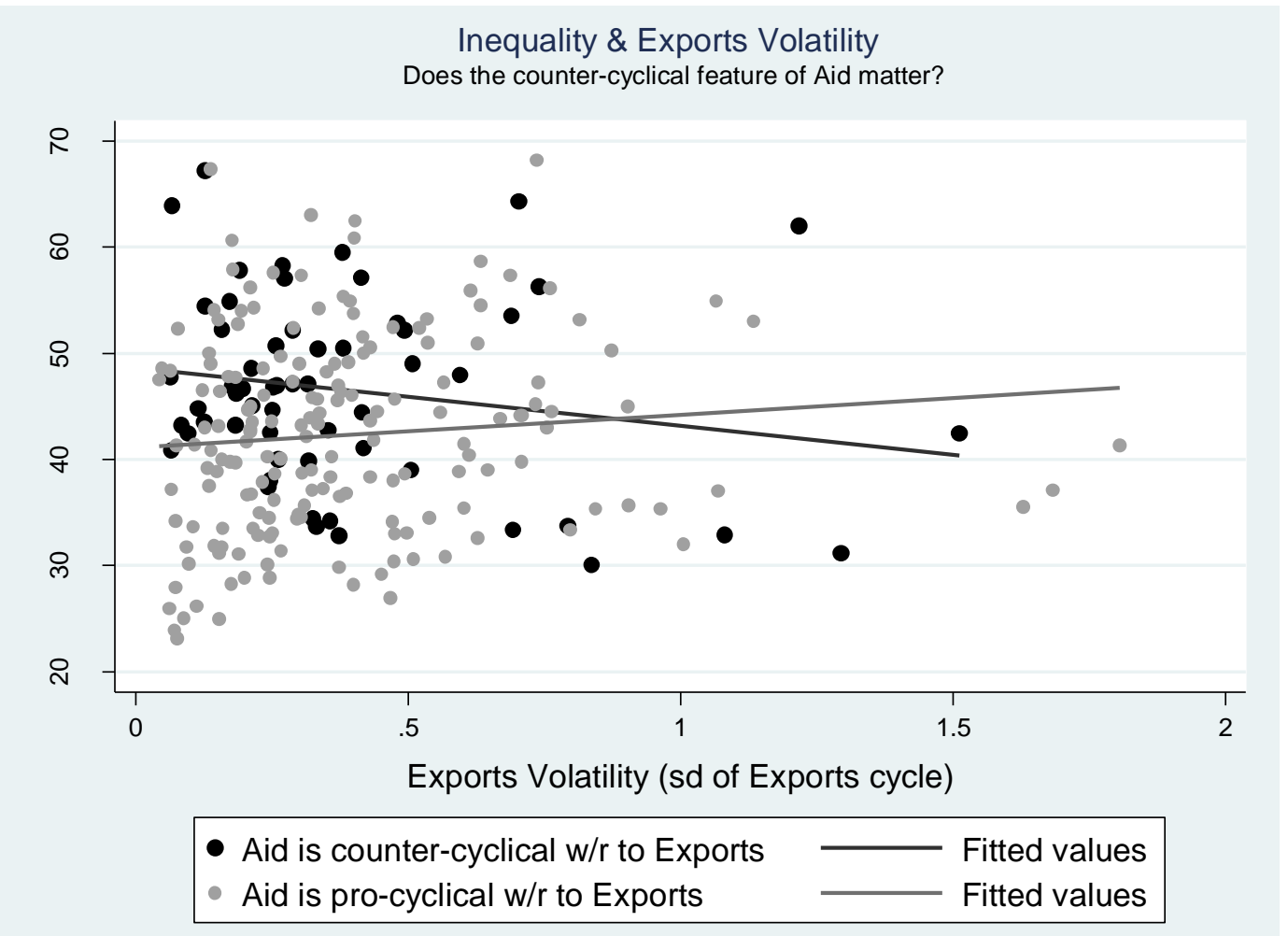


In order to examine whether aid or remittances decrease output volatility by mitigating the destabilizing impact of exports instability, we build on Chauvet and Guillaumont (2009) and estimate the following model:

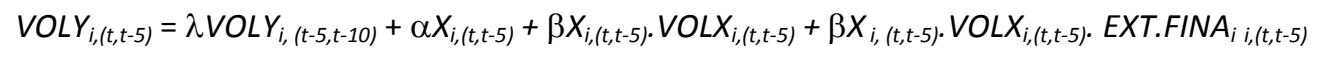

$$
\begin{aligned}
& +\gamma{\operatorname{EXT} . F I N A_{i, j, t, t-5)}}+\delta \text { EXT.FINA }_{i,(t, t-5)} \times \operatorname{VOLEXT.FINA~}_{i,(t, t-5)}+\varpi X_{i,(t, t-5)}+\mu_{i}+\tau_{t}+\varepsilon_{i,(t, t-5)}
\end{aligned}
$$

where $X_{i, t, t,-5)}$ stands for exports of goods and services over GDP, averaged over $t$ and $t-5$, and $V O L X_{i,(t,-5)}$ is the volatility of exports, and as before EXT.FINA $A_{i, t(t,-5)}$ represents foreign aid and remittances as a percentage of GDP. The volatility of external financial flows and exports is measured in the same way as the volatility of income per capita (see Section 2), by a series in constant US dollars, deflated using US unit import prices. The volatility of income per capita is a function of the volatility of exports weighted by the size of exports (exports in GDP, as a proxy for the exposure of the economy to export volatility), and controlling for exports in GDP. Aid and remittances as a function of GDP may directly decrease income volatility. However, the volatility of aid, and of remittances to a lower extent, may be an additional source of external volatility, which is more pervasive in countries highly dependent on these flows. Aid and remittances volatility is therefore weighted by the share of each flow in GDP. We control for a set of country characteristics (initial income volatility, inflation rate, GDP per capita and its square, total and rural population, secondary school enrolment, government expenditures). This Chauvet and Guillaumont (2009) model is augmented with a triple interaction term of export volatility weighted by the share of exports of GDP and multiplied by aid (and remittances). A negative coefficient of this variable would indicate that aid (or remittances) dampens the output volatility inducing effect of export instability. Table 9 presents the results. We use both the full sample of countries and a restricted sample of only developing countries.

In line with Chauvet and Guillaumont (2009), the results show that export volatility tends to increase income fluctuations, especially when the country is largely open. However, we find little evidence that aid volatility plays a similar role on income volatility. Aid volatility leads to more income instability only when an extra set of control variables is added and only when the whole sample is considered: on the sample of only developing countries aid volatility is no longer significant. Moreover, income volatility does not seem to be characterized by high persistence, since the lagged dependent variable is never significant across the different specifications.

Turning to our variable of interest, the results show a negative and significant coefficient for the triple interaction variable (exports, volatility of exports, aid) which is robust across the different control variable sets and samples considered. Although, the magnitude of the coefficient remains rather small, this shows that aid significantly reduces income volatility in larger exporting countries with big export volatility. This result therefore highlights the role played by international aid to reduce the adverse effects of export volatility on income fluctuations, and highlights one of the channels by which aid reduces income inequalities. 
Looking next at the results for remittances, we do not observe any statistically significant effect of the triple interaction variable (exports, volatility of exports, remittances) on income volatility. However, in line with the literature, Table 10 shows that remittances can potentially directly reduce income volatility, since the coefficient of their measure in level is negative and significant in columns 1, 2 and 5 . It should be noted that this result appears to be less robust than the one for aid and its triple interaction; moreover it does not allow us to conclude that there is a positive contribution of remittances on inequality reduction through its stabilizing effect on income volatility, because remittances volatility displays a strong and significant effect on income volatility. Hence, while the level of remittances tends to lower income volatility, its own volatility may have harmful effects on inequality by making economic growth less stable. 
Table 9: Income volatility and Aid, Sys-GMM, 1973-2012, 5-year periods.

\begin{tabular}{|c|c|c|c|c|c|c|c|c|}
\hline \multirow[b]{2}{*}{$\begin{array}{l}\text { Sys-GMM - Internal } \\
\text { instruments }\end{array}$} & \multicolumn{7}{|c|}{ Dependent variables: GDP per capita volatility } & \multirow[b]{2}{*}{8} \\
\hline & 1 & 2 & 3 & 4 & 5 & 6 & 7 & \\
\hline & \multicolumn{4}{|c|}{ All countries } & \multicolumn{4}{|c|}{ Developing countries only } \\
\hline Ratio of ODA to GDP & $\begin{array}{l}-0.008 \\
(0.006)\end{array}$ & $\begin{array}{l}-0.003 \\
(0.014)\end{array}$ & $\begin{array}{l}-0.005 \\
(0.020)\end{array}$ & $\begin{array}{c}0.019 \\
(0.018)\end{array}$ & $\begin{array}{l}-0.009 \\
(0.008)\end{array}$ & $\begin{array}{c}0.001 \\
(0.013)\end{array}$ & $\begin{array}{c}0.022 \\
(0.024)\end{array}$ & $\begin{array}{l}-0.009 \\
(0.009)\end{array}$ \\
\hline $\begin{array}{l}\text { Ratio of ODA to GDP x ODA } \\
\text { volatility }\end{array}$ & $\begin{array}{l}0.003 \\
(0.004)\end{array}$ & $\begin{array}{c}0.020^{* * *} \\
(0.006)\end{array}$ & $\begin{array}{c}0.000 \\
(0.004)\end{array}$ & $\begin{array}{l}-0.003 \\
(0.006)\end{array}$ & $\begin{array}{l}0.006 \\
(0.004)\end{array}$ & $\begin{array}{l}-0.000 \\
(0.007)\end{array}$ & $\begin{array}{c}0.003 \\
(0.008)\end{array}$ & $\begin{array}{l}-0.003 \\
(0.011)\end{array}$ \\
\hline No ODA dummy & $\begin{array}{l}0.044 \\
(0.086)\end{array}$ & $\begin{array}{l}-0.332+ \\
(0.207)\end{array}$ & $\begin{array}{c}0.461 \\
(0.512)\end{array}$ & $\begin{array}{l}-0.185 \\
(0.183)\end{array}$ & $\begin{array}{l}0.078 \\
(0.129)\end{array}$ & $\begin{array}{c}0.043 \\
(0.327)\end{array}$ & $\begin{array}{c}0.076 \\
(0.453)\end{array}$ & $\begin{array}{l}-0.090 \\
(0.296)\end{array}$ \\
\hline Ratio of Exports to GDP & $\begin{array}{c}0.002 \\
(0.001)\end{array}$ & $\begin{array}{l}0.003 \\
(0.004)\end{array}$ & $\begin{array}{c}0.001 \\
(0.002)\end{array}$ & $\begin{array}{c}0.002 \\
(0.003)\end{array}$ & $\begin{array}{c}0.000 \\
(0.002)\end{array}$ & $\begin{array}{l}-0.006^{*} \\
(0.003)\end{array}$ & $\begin{array}{l}-0.001 \\
(0.010)\end{array}$ & $\begin{array}{l}-0.006 \\
(0.006)\end{array}$ \\
\hline $\begin{array}{l}\text { Ratio of Exports to GDP x } \\
\text { Exports volatility }\end{array}$ & $\begin{array}{c}0.005^{* * *} \\
(0.002)\end{array}$ & $\begin{array}{l}-0.006 \\
(0.011)\end{array}$ & $\begin{array}{l}0.006^{* *} \\
(0.002)\end{array}$ & $\begin{array}{l}-0.000 \\
(0.006)\end{array}$ & $\begin{array}{l}0.003+ \\
(0.002)\end{array}$ & $\begin{array}{l}0.006^{* *} \\
(0.003)\end{array}$ & $\begin{array}{c}0.017 \\
(0.021)\end{array}$ & $\begin{array}{l}0.005^{*} \\
(0.003)\end{array}$ \\
\hline $\begin{array}{l}\text { Exports (over GDP) } x \\
\text { Exports volatility x ODA } \\
\text { (over GDP) }\end{array}$ & & & $\begin{array}{l}-0.001 * \\
(0.001)\end{array}$ & $\begin{array}{c}-0.001 * \\
(0.001)\end{array}$ & & & $\begin{array}{c}-0.002 * * \\
(0.001)\end{array}$ & $\begin{array}{c}0.001 \\
(0.001)\end{array}$ \\
\hline Lagged dependent & $\begin{array}{c}0.111 \\
(0.101)\end{array}$ & $\begin{array}{l}0.032 \\
(0.102)\end{array}$ & $\begin{array}{c}0.088 \\
(0.124)\end{array}$ & $\begin{array}{c}0.070 \\
(0.071)\end{array}$ & $\begin{array}{c}0.123 \\
(0.117)\end{array}$ & $\begin{array}{c}0.224 \\
(0.195)\end{array}$ & $\begin{array}{l}-0.031 \\
(0.115)\end{array}$ & $\begin{array}{c}0.239 \\
(0.233)\end{array}$ \\
\hline GDP per capita (in log) & $\begin{array}{c}-0.160^{* *} \\
(0.081)\end{array}$ & $\begin{array}{l}-0.012 \\
(0.240)\end{array}$ & $\begin{array}{l}-0.291 \\
(0.208)\end{array}$ & $\begin{array}{l}-0.079 \\
(0.200)\end{array}$ & $\begin{array}{l}-0.167^{*} \\
(0.099)\end{array}$ & $\begin{array}{l}-0.799 \\
(0.636)\end{array}$ & $\begin{array}{l}-0.259^{* *} \\
(0.127)\end{array}$ & $\begin{array}{l}-1.068^{*} \\
(0.633)\end{array}$ \\
\hline $\begin{array}{l}\text { GDP per capita squared (in } \\
\text { log) }\end{array}$ & & $\begin{array}{l}0.005 \\
(0.014)\end{array}$ & & $\begin{array}{c}0.006 \\
(0.013)\end{array}$ & & $\begin{array}{c}0.055 \\
(0.044)\end{array}$ & & $\begin{array}{l}0.072+ \\
(0.045)\end{array}$ \\
\hline Population growth & & $\begin{array}{l}-0.034 \\
(0.035)\end{array}$ & & $\begin{array}{l}-0.017 \\
(0.038)\end{array}$ & & $\begin{array}{c}0.055 \\
(0.094)\end{array}$ & & $\begin{array}{c}0.052 \\
(0.082)\end{array}$ \\
\hline Rural population (in log) & & $\begin{array}{l}-0.045 \\
(0.043)\end{array}$ & & $\begin{array}{l}-0.031 \\
(0.033)\end{array}$ & & $\begin{array}{l}-0.097 \\
(0.090)\end{array}$ & & $\begin{array}{l}-0.108 \\
(0.100)\end{array}$ \\
\hline Inflation (in log) & & $\begin{array}{l}0.033 \\
(0.033)\end{array}$ & & $\begin{array}{c}0.034 \\
(0.026)\end{array}$ & & $\begin{array}{c}0.015 \\
(0.025)\end{array}$ & & $\begin{array}{c}0.014 \\
(0.031)\end{array}$ \\
\hline $\begin{array}{l}\text { Secondary school } \\
\text { enrollment (gross, in log) }\end{array}$ & & $\begin{array}{l}-0.164^{*} \\
(0.088)\end{array}$ & & $\begin{array}{l}-0.090 \\
(0.097)\end{array}$ & & $\begin{array}{c}0.036 \\
(0.116)\end{array}$ & & $\begin{array}{c}0.045 \\
(0.137)\end{array}$ \\
\hline $\begin{array}{l}\text { Ratio of Government } \\
\text { expenditure to GDP (in log) }\end{array}$ & & $\begin{array}{l}0.040 \\
(0.133)\end{array}$ & & $\begin{array}{c}0.035 \\
(0.079)\end{array}$ & & $\begin{array}{c}0.015 \\
(0.086)\end{array}$ & & $\begin{array}{l}-0.015 \\
(0.119)\end{array}$ \\
\hline Number of observations & 642 & 483 & 642 & 483 & 447 & 329 & 447 & 329 \\
\hline Number of countries & 174 & 155 & 174 & 155 & 118 & 102 & 118 & 102 \\
\hline Developing countries only & No & No & No & No & Yes & Yes & Yes & Yes \\
\hline AR1 (p-value) & 0.487 & 0.037 & 0.260 & 0.005 & 0.564 & 0.122 & 0.125 & 0.141 \\
\hline AR2 ( $p$-value) & 0.329 & 0.354 & 0.603 & 0.303 & 0.263 & 0.249 & 0.352 & 0.163 \\
\hline Hansen test (p-value) & 0.237 & 0.702 & 0.356 & 0.683 & 0.871 & 0.331 & 0.689 & 0.273 \\
\hline
\end{tabular}

Notes: Robust standard errors in parentheses (using the Windmeijer correction), $+p<0.15,{ }^{*} p<0.10,{ }^{* *} p<0.05$, *** $p<0.01$. Each specification includes period dummies and a constant.

Sources: Authors' calculations based on UNU-WIDER, World Bank and OECD data. 
Table 10: Income volatility and Remittances, Sys-GMM, 1973-2012, 5-year periods.

\begin{tabular}{|c|c|c|c|c|c|c|c|c|}
\hline \multicolumn{9}{|c|}{ Dependent variables: GDP per capita volatility } \\
\hline \multirow[t]{2}{*}{$\begin{array}{l}\text { Sys-GMM - Internal } \\
\text { instruments }\end{array}$} & 1 & 2 & 3 & 4 & 5 & 6 & 7 & 8 \\
\hline & \multicolumn{4}{|c|}{ All countries } & \multicolumn{4}{|c|}{ Developing countries only } \\
\hline Ratio of Remittances to GDP & $\begin{array}{l}-0.008^{* *} \\
(0.004)\end{array}$ & $\begin{array}{l}-0.006^{*} \\
(0.003)\end{array}$ & $\begin{array}{l}-0.005 \\
(0.007)\end{array}$ & $\begin{array}{l}-0.003 \\
(0.008)\end{array}$ & $\begin{array}{c}-0.009^{* *} \\
(0.004)\end{array}$ & $\begin{array}{l}-0.004 \\
(0.003)\end{array}$ & $\begin{array}{l}-0.004 \\
(0.008)\end{array}$ & $\begin{array}{l}-0.001 \\
(0.008)\end{array}$ \\
\hline Ratio of Remittances to GDP $x$ & $0.848^{* *}$ & $1.118^{* * *}$ & $1.003^{* * *}$ & $1.147^{* * * *}$ & $1.491^{* * *}$ & $0.982^{* *}$ & $1.456^{* * *}$ & $0.944^{* *}$ \\
\hline emittance & $(0.411)$ & $(0.396)$ & $(0.379)$ & $(0.380)$ & $(0.513)$ & $(0.380)$ & $(0.525)$ & $(0.372)$ \\
\hline Ratio of Expo & $\begin{array}{c}0.001 \\
(0.001)\end{array}$ & $\begin{array}{l}-0.001 \\
(0.001)\end{array}$ & $\begin{array}{c}0.001 \\
(0.001)\end{array}$ & $\begin{array}{l}-0.001 \\
(0.001)\end{array}$ & $\begin{array}{l}-0.001 \\
(0.002)\end{array}$ & $\begin{array}{l}-0.002 \\
(0.002)\end{array}$ & $\begin{array}{l}-0.002 \\
(0.002)\end{array}$ & $\begin{array}{l}-0.001 \\
(0.002)\end{array}$ \\
\hline $\begin{array}{l}\text { Ratio of Exports to GDP x } \\
\text { Exports volatility }\end{array}$ & $\begin{array}{c}0.004 \\
(0.003)\end{array}$ & $\begin{array}{c}0.002 \\
(0.002)\end{array}$ & $\begin{array}{l}0.004+ \\
(0.003)\end{array}$ & $\begin{array}{l}0.004^{*} \\
(0.002)\end{array}$ & $\begin{array}{c}0.003 \\
(0.002)\end{array}$ & $\begin{array}{l}0.004+ \\
(0.002)\end{array}$ & $\begin{array}{c}0.003 \\
(0.002)\end{array}$ & $\begin{array}{l}0.004+ \\
(0.003)\end{array}$ \\
\hline $\begin{array}{l}\text { Ratio of Exports to GDP } x \\
\text { Exports volatility } x \text { Ratio of } \\
\text { Remittances to GDP }\end{array}$ & & & $\begin{array}{l}-0.001 \\
(0.001)\end{array}$ & $\begin{array}{l}-0.001 \\
(0.001)\end{array}$ & & & $\begin{array}{l}-0.001 \\
(0.001)\end{array}$ & $\begin{array}{l}-0.001 \\
(0.001)\end{array}$ \\
\hline -agged dependent & $\begin{array}{c}0.037 \\
(0.043)\end{array}$ & $\begin{array}{c}0.021 \\
(0.041)\end{array}$ & $\begin{array}{c}0.021 \\
(0.042)\end{array}$ & $\begin{array}{l}0.016 \\
(0.037)\end{array}$ & $\begin{array}{l}0.079 \\
(0.056)\end{array}$ & $\begin{array}{l}0.128^{* *} \\
(0.063)\end{array}$ & $\begin{array}{c}0.073 \\
(0.061)\end{array}$ & $\begin{array}{l}0.118^{*} \\
(0.061)\end{array}$ \\
\hline GDP per & $\begin{array}{c}-0.112^{* * *} \\
(0.033)\end{array}$ & $\begin{array}{l}-0.180 \\
(0.132)\end{array}$ & $\begin{array}{l}-0.105^{* * *} \\
(0.032)\end{array}$ & $\begin{array}{r}-0.209+ \\
(0.129)\end{array}$ & $\begin{array}{c}0.019 \\
(0.039)\end{array}$ & $\begin{array}{l}-0.430 \\
(0.310)\end{array}$ & $\begin{array}{l}0.021 \\
(0.032)\end{array}$ & $\begin{array}{l}-0.488^{*} \\
(0.280)\end{array}$ \\
\hline $\begin{array}{l}\text { GDP per capita squared (in } \\
\text { log) }\end{array}$ & & $\begin{array}{c}0.012 \\
(0.017)\end{array}$ & & $\begin{array}{c}0.011 \\
(0.017)\end{array}$ & & $\begin{array}{l}0.008 \\
(0.021)\end{array}$ & & $\begin{array}{c}0.007 \\
(0.021)\end{array}$ \\
\hline Population growth & & $\begin{array}{l}-0.037 \\
(0.046)\end{array}$ & & $\begin{array}{l}-0.031 \\
(0.048)\end{array}$ & & $\begin{array}{l}-0.059 \\
(0.048)\end{array}$ & & $\begin{array}{l}-0.052 \\
(0.049)\end{array}$ \\
\hline Rural & & $\begin{array}{c}0.112^{* * *} \\
(0.040)\end{array}$ & & $\begin{array}{c}0.111^{* * *} \\
(0.041)\end{array}$ & & $\begin{array}{l}0.109^{* *} \\
(0.046)\end{array}$ & & $\begin{array}{l}0.109^{* *} \\
(0.047)\end{array}$ \\
\hline Inflation (in log) & & $\begin{array}{l}0.007 \\
(0.007)\end{array}$ & & $\begin{array}{c}0.009 \\
(0.007)\end{array}$ & & $\begin{array}{c}0.027 \\
(0.020)\end{array}$ & & $\begin{array}{l}0.030+ \\
(0.018)\end{array}$ \\
\hline $\begin{array}{l}\text { Secondary school enrollment } \\
\text { gross, in log) }\end{array}$ & & $\begin{array}{l}-0.022 \\
(0.016)\end{array}$ & & $\begin{array}{c}-0.025+ \\
(0.016)\end{array}$ & & $\begin{array}{l}-0.025 \\
(0.023)\end{array}$ & & $\begin{array}{l}-0.027 \\
(0.022)\end{array}$ \\
\hline $\begin{array}{l}\text { Ratio of Government } \\
\text { expenditure to GDP (in log) }\end{array}$ & & $\begin{array}{c}-0.033+ \\
(0.022)\end{array}$ & & $\begin{array}{c}-0.034+ \\
(0.022)\end{array}$ & & $\begin{array}{l}-0.066 \\
(0.049)\end{array}$ & & $\begin{array}{l}-0.070 \\
(0.049)\end{array}$ \\
\hline Number of observations & 504 & 411 & 50 & 411 & 35 & 278 & 353 & 278 \\
\hline Vumb & 158 & 143 & $15 \varepsilon$ & 17 & 10 & 95 & 108 & 95 \\
\hline Developing countries only & No & No & No & No & Yes & Yes & Yes & Yes \\
\hline AR1 (p-value) & 0.002 & 0.003 & 0.001 & 0.002 & 0.003 & 0.122 & 0.002 & 0.102 \\
\hline AR2 (p-value) & 0.808 & 0.331 & 0.979 & 0.391 & 0.868 & 0.912 & 0.989 & 0.802 \\
\hline Hansen test (p-value) & 0.756 & 0.117 & 0.799 & 0.130 & 0.811 & 0.181 & 0.608 & 0.318 \\
\hline
\end{tabular}

Notes: Robust standard errors in parentheses (using the Windmeijer correction), $+p<0.15,{ }^{*} p<0.10,{ }^{* *} p<0.05$, *** $\mathrm{p}<0.01$. Each specification includes period dummies and a constant.

Sources: Authors' calculations based on UNU-WIDER, World Bank and OECD data. 


\section{Conclusions}

While income growth is a major factor in poverty reduction, income volatility hurts the poor through its negative effect on income growth. If macroeconomic volatility generates inequality, and if aid or remittances have a stabilizing impact, it should be expected that due to this impact they contribute to poverty reduction not only by increasing the rate of growth but also by making this growth less volatile and more pro-poor, by mitigating the adverse effect of volatility on income distribution.

In this paper, we test the hypothesis that foreign aid and remittances may dampen the adverse effect of macroeconomic volatility on income inequality. We find that volatility has a robust and positive impact on inequality, and that aid tends to reduce volatility and simultaneously to dampen its positive impact on inequality (i.e. negative impact on the poor). The effect of remittances is more uncertain.

In addition, we suggest two main channels through which aid affects inequality. First, aid can support increases in public expenditures in favour of the poor during negative shocks. Our evidence suggests that it prevents the poorest taking their children out of school, thus securing their investment in human capital, and reducing income inequality in the long-run. The second potential channel, highlighted above, is that aid can reduce inequality through its direct stabilizing effect on income volatility. Our results tend to show that aid reduces income volatility in countries exposed to high levels of export volatility.

The contribution of remittances is less clear. Although we observe at first that remittances tend to mitigate the negative impact of income volatility on inequality, these results were not robust once we controlled for endogeneity and persistency in our dependent variable. Our results indicate that remittances do not seem to influence income inequality through a mitigating or stabilizing effect on income volatility. 


\section{Appendix}

Table A0: Sample of countries

\begin{tabular}{|c|c|c|c|c|c|}
\hline Country Name & Freq. & Country Name & Freq. & Country Name & Freq. \\
\hline Afghanistan & 2 & Gambia, The & 2 & Nicaragua & 2 \\
\hline Albania & 4 & Georgia & 2 & Niger & 2 \\
\hline Algeria & 2 & Germany & 5 & Nigeria & 2 \\
\hline Angola & 2 & Ghana & 3 & Norway & 7 \\
\hline Armenia & 4 & Greece & 7 & Pakistan & 4 \\
\hline Australia & 4 & Guatemala & 5 & Panama & 6 \\
\hline Austria & 7 & Guinea & 1 & Papua New Guinea & 1 \\
\hline Azerbaijan & 2 & Guinea-Bissau & 1 & Paraguay & 5 \\
\hline Bahamas, The & 5 & Guyana & 1 & Peru & 7 \\
\hline Bangladesh & 5 & Honduras & 5 & Philippines & 6 \\
\hline Barbados & 2 & Hong Kong, China & 3 & Poland & 4 \\
\hline Belarus & 3 & Iceland & 2 & Portugal & 6 \\
\hline Belgium & 7 & India & 5 & Qatar & 1 \\
\hline Belize & 2 & Indonesia & 4 & Romania & 5 \\
\hline Benin & 1 & Iran, Islamic Rep. & 4 & Russian Federation & 4 \\
\hline Bhutan & 1 & Iraq & 1 & Rwanda & 3 \\
\hline Bolivia & 3 & Ireland & 6 & Senegal & 2 \\
\hline Botswana & 3 & Israel & 7 & Serbia & 3 \\
\hline Bulgaria & 6 & Italy & 7 & Seychelles & 2 \\
\hline Burkina Faso & 2 & Japan & 5 & Slovak Republic & 4 \\
\hline Burundi & 3 & Jordan & 4 & Slovenia & 3 \\
\hline Cambodia & 3 & Kazakhstan & 2 & South Africa & 5 \\
\hline Cameroon & 1 & Kenya & 3 & Spain & 7 \\
\hline Canada & 7 & Korea, Rep. & 7 & Sri Lanka & 3 \\
\hline Central African Republic & 1 & Kyrgyz Republic & 4 & St. Lucia & 1 \\
\hline Chile & 1 & Lao PDR & 3 & Sudan & 1 \\
\hline China & 4 & Latvia & 2 & Suriname & 1 \\
\hline Colombia & 7 & Lesotho & 3 & Swaziland & 3 \\
\hline Comoros & 1 & Lithuania & 1 & Sweden & 7 \\
\hline Congo, Dem. Rep. & 1 & Luxembourg & 6 & Switzerland & 4 \\
\hline Congo, Rep. & 1 & Macedonia, FYR & 4 & Syrian Arab Republic & 1 \\
\hline Costa Rica & 7 & Madagascar & 2 & Tajikistan & 3 \\
\hline Cote d'Ivoire & 2 & Malawi & 5 & Tanzania & 2 \\
\hline Croatia & 3 & Malaysia & 6 & Thailand & 7 \\
\hline Cyprus & 3 & Mali & 4 & Togo & 1 \\
\hline Czech Republic & 4 & Malta & 3 & Trinidad and Tobago & 2 \\
\hline Denmark & 7 & Mauritania & 5 & Tunisia & 5 \\
\hline Dominican Republic & 5 & Mauritius & 6 & Turkey & 6 \\
\hline East Timor & 1 & Mexico & 6 & Uganda & 3 \\
\hline Ecuador & 5 & Moldova & 4 & Ukraine & 3 \\
\hline Egypt, Arab Rep. & 4 & Mongolia & 3 & United Kingdom & 5 \\
\hline El Salvador & 5 & Montenegro & 2 & United States & 7 \\
\hline Estonia & 3 & Morocco & 5 & Uruguay & 7 \\
\hline Ethiopia & 3 & Mozambique & 3 & Venezuela & 1 \\
\hline Fiji & 3 & Namibia & 1 & Vietnam & 1 \\
\hline Finland & 7 & Nepal & 3 & West Bank and Gaza & 2 \\
\hline France & 7 & Netherlands & 7 & & \\
\hline Gabon & 1 & New Zealand & 6 & Total & 520 \\
\hline
\end{tabular}




\section{References}

- Adams R.H. and J.Page (2005) «Do international migration and remittances reduce poverty in developing countries? World Development, Volume 33, Issue 10, pages 1645-1669.

- Agénor, P_R., (2002) Business Cycles, Economic Crises and the Poor: Testing for Asymmetric Effects, Journal of Policy Reform, 5 (oct.) : 145-160.

- Agénor, P-R., (2004). Macroeconomic Adjustment and the Poor: Analytical Issues and Cross-Country Evidence, Journal of Economic Surveys 18(3): 351-408.

- Atkinson, A. B. and Brandolini, A. (2001). Promise and pitfalls in the use of "secondary" data-sets: income inequality in OECD countries as a case study, Journal of Economic Literature, 34 (3): 771-799.

- Atkinson, A. B. and Brandolini, A. (2009). On data: a case study of the evolution of income inequality across time and across countries, Cambridge Journal of Economics, 33 (3): 381-404.

- or FDI, Journal of Economic Integration 31(1), 65-102.

- Barham B. and S. Boucher (1998). Migration, remittances, and inequality: estimating the net effects of migration on income distribution Journal of Development Economics, Volume 55, Issue 2, pages 307-331

- Bjornskov, C (2010). Do elites benefit from democracy and foreign aid in developing countries? Journal of Development Economics, 92:115-24.

- Boone, P., (1996). Politics and the effectiveness of foreign aid. European Economic Review 40, 289-329.

- Bouoiyour J., R. Selmi, and A. Miftah (2016), What Mitigates Economic Growth Volatility in Morocco? : Remittances Breen, R. and C. Garcia-Penalosa (2005). Income Inequality and Income volatility: An Empirical Investigation. Review of Development Economics, 9(3): 380-98.
- Bugamelli M., and F. Paternò (2011), Output Growth Volatility and Remittances, Economica 78, 480-500.

- Bulír, A. and A.J. Hamann, (2001). "How Volatile and Unpredictable Are Aid Flows, and What Are the Policy Implications?" IMF working paper 167, Washington D.C.

- Bulír, Ales and Hamann, A. Javier, (2008). Volatility of Development Aid: From the Frying Pan into the Fire?, World Development, 36(10): 2048-2066.

- Calderón, C., A. Chong, and M. Gradstein (2009). Can foreign aid reduce income inequality and poverty? Public Choice. Vol140. pp 59-84.

- Calderón, C., and Levy Yeyati, E. L., (2009). Zooming In: From Aggregate Volatility to Income Distribution. Policy Research Working Paper, World Bank.

- Chase-Dunn, C., (1975). The effects of international economic dependence on development and inequality: a crossnational study. American Sociological Review 40, 720-738.

- Chauvet, L. and P. Guillaumont (2004). Aid and Growth Revisited : Policy, Economic Vulnerability and Political Instability. In B. Tungodden, N. Stern and I. Koldstad (eds.), Towards Pro-Poor Policies - Aid, Institutions and Globalization. ABCDE 2003 Annual World Bank Conference on Development Economics Europe (New York: Oxford University Press).

- Chauvet L. and P. Guillaumont (2009). Aid, Volatility and Growth Again. When Aid Volatility Matters and When it Does Not, Review of Development Economics, (13), 3, August, 452-463

- Chauvet, L. and Mesplé-Somps, S. (2007). Impact des financements internationaux sur les inégalités des pays en développement, Revue Economique 2007/3 vol 58, 735-744.

- Chen, S., and M. Ravallion (2008). 'The Developing World is Poorer than We 
Thought, But No Less Successful in the Fight against Poverty'. Poverty Research Paper 4703. Washington, DC: World Bank.

- Combes J-L., Ebeke C.H. and M. Ntsama Etoundi (2014). Are Remittances and Foreign Aid a Hedge Against Food Price Shocks in Developing Countries? World Development Vol. 54 p. 81-98

- De S., E. Islamaj, M. A. Kose, S. R. Yousefi (2016), Remittances over the Business Cycle: Theory and Evidence, CAMA Working Paper 13/2016.

- Deininger, K., and L., Squire (1996). A New Data Set Measuring Income Inequality. World Bank Economic Review 10(3):565591.

- Dercon, S. and P. Krishnan (2000). In Sickness and in Health: Risk Sharing Within Households in Rural Ethiopia. Journal of Political Economy, 108(4): 688-727.

- Ebeke C.H. and M. Le Goff (2009). Why Migrants' Remittances Reduce Income Inequality in some Countries and not in Others? CERDI Etudes et documents, 2009 $\mathrm{n}^{\circ} 19$

- Guillaumont P. (2006), Macro vulnerability in low income countries and aid responses, in Securing Development in an Unstable Word (F. Bourguignon, B. Pleskovic, and J. van der Gaag, Edrs) ABCDE Europe 2005, Word Bank, 65-108

- Guillaumont P. (2009). Caught in a trap. Identifying the least developed countries, Economica

- Guillaumont, P., S. Guillaumont Jeanneney, and J.-F. Brun (1999). How Instability Lowers African Growth. Journal of African Economies, 8(1): 87-107.

- Guillaumont, P. and L. Chauvet (2001). Aid and performance: A reassessment. Journal of Development Studies, 37(6): 66-92.

- Guillaumont, P. and M. Le Goff, (2010). Aid and Remittances: Their Stabilizing Impact Compared, FERDI working paper $\mathrm{n}^{\circ} 12$.
- Guillaumont, P. and Wagner, L. (2012). Aid and Growth Accelerations: Vulnerability Matters, Working Papers UNU-WIDER Research Paper, World Institute for Development Economic Research (UNUWIDER).

- Guillaumont, P., Wagner, L., 2013, « Aid effectiveness for poverty reduction: macroeconomic overview and emerging issues ", Revue d'Economie du Développement, Issue 4, Vol 2, pp 115-164.

- Guillaumont Jeanneney, S. and K. Kpodar (2011). Financial Development and Poverty Reduction: Can There be a Benefit without a Cost? Journal of Development Studies, Taylor and Francis Journals, vol. 47(1), pages 143-163.

- Herzer, D, P Nunnenkamp (2012). The effect of foreign aid on income inequality: Evidence from panel cointegration, Working Paper 1762. Kiel Institute for the World Economy, Kiel.

- Hnatkovska, V. and N. Loayza (2005). Volatility and Growth. In J. Aizenmann and B. Pinto (eds), Managing Volatility and Crises: A Practitioner s Guide. World Bank, Cambridge University Press.

- Laursen, T. and S. Mahajan (2005). Volatility, Income Distribution, and Poverty. In J. Aizen- man and B. Pinto (eds), Managing Volatility and Crisis: A Practitioner Guide, The World Bank, Cambridge University Press.

- Le Goff M. (2010). How Remittances Contribute to Poverty Reduction: a Stabilizing Effect, CERDI WP Etudes et documents, $2010 \mathrm{n}^{\circ} 8$

- Le Goff M. (2010). Migrant remittances, foreign aid and development of recipient countries, these soutenue à l'Université d'Auvergne le 29/03/2012.

- Milanovic, S. (2013). Description of All The Ginis Dataset. World Bank, Research Department. 
- Norrbin, S. C. and F. Pinar Yigit (2005). The Robustness of the Link Between Volatility and Growth of Output. Review of World Economics, 141 (2): 343-56.

- Pallage, S. and M.A. Robe, (2001). Foreign Aid and the Business Cycle, Review of International Economics 9(4): 641-72.

- Raddatz, C., (2007). Are External Shocks Responsible for the instability of output in low-income countries? Journal of Development Economics 84: 155-187.

- Ramey, G. and Ramey, V. A. (1995). Cross country evidence on the link between Volatility and Growth, American Economic Review, 85(5): 1138-1151.

- Rapoport H. and B. Docquier (2006). The Economics of Migrants' Remittances, Handbook of the Economics of Giving, Altruism and Reciprocity. Applications, Volume 2, 2006, Chapter 17 Pages 11351198.
- An introduction to difference and system GMM in Stata. Center for Global Development working paper, (103).

- Stark O. Taylor J.E. and S. Yitzhaki (1988). Migration, remittances and inequality: $\mathrm{A}$ sensitivity analysis using the extended Gini index, Journal of Development Economics, Volume 28, Issue 3, pages 309322Tavares, J. (2003). Does foreign aid corrupt? Economics Letters, 79(1), 99-106.

- Taylor J. E. (1992). Remittances and inequality reconsidered: Direct, indirect, and intertemporal effects, Journal of Policy Modeling, Volume 14, Issue 2, pages 187208

- Thomas, D., K. Beegle, E. Frankenberg, B. Sikoki, J.Strauss and G. Teruel (2004). Education in a Crisis". Journal of Development Economics, 74(1): 53-85. 

"Sur quoi la fondera-t-il l'économie du monde qu'il veut gouverner? Sera-ce sur le caprice de chaque particulier? Quelle confusion! Sera-ce sur la justice? Il l'ignore."

Pascal

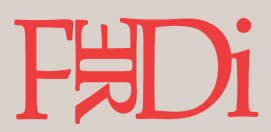

Created in 2003, the Fondation pour les études et recherches sur le développement international aims to promote a fuller understanding of international economic development and the factors that influence it.

\section{$\hookrightarrow$ Contact}

www.ferdi.fr

contact@ferdi.fr

+33 (o)4 73177530 